Check for updates

Cite this: RSC Adv., 2017, 7, 25031

Received 2nd March 2017

Accepted 2nd May 2017

DOI: 10.1039/c7ra02574b

rsc.li/rsc-advances

\section{Palladium(II)-catalyzed ortho-C-H olefination of phenylalanine and phenylethylamine derivatives directed by removable picolinamide group $\uparrow$}

\author{
Fei Zhao, iD *a Xiuwen Jia, ${ }^{a}$ Jingwei Zhao, ${ }^{a}$ Chaoli Fei, ${ }^{a}$ Liyang Liu, ${ }^{a}$ Guannan Liu, \\ Dongping Wang ${ }^{a}$ and Fei Chen ${ }^{a}$
}

Palladium-catalyzed ortho- $\mathrm{C}-\mathrm{H}$ olefination of phenylalanine and phenylethylamine derivatives assisted by a removable picolinamide group has been achieved. This protocol shows broad substrate scope, functional tolerance and moderate to high yields, thus affording a practical approach for the direct modification of phenylalanine and phenylethylamine derivatives.

\section{Introduction}

Transition-metal-catalyzed direct $\mathrm{C}-\mathrm{H}$ functionalization has captured wide attention because of its high efficiency in constructing carbon-carbon and carbon-heteroatom bonds, ${ }^{1,2}$ and great progress has been made in $\mathrm{C}-\mathrm{H}$ activation reactions with the assistance of various directing groups which can enhance the reactivity and achieve site selectivity in recent decades. ${ }^{3,4}$ In particular, $\mathrm{C}-\mathrm{H}$ functionalization of nitrogen-containing compounds, such as amino acid derivatives, is especially important and attractive considering their prevalence in natural products and therapeutic agents. Moreover, functionalized amino acid derivatives have also been widely used as building blocks in medicinal chemistry for their broad biological activities. ${ }^{5}$ Therefore, the synthesis of these structures is of great importance. Compared with the conventional de novo synthesis strategy, the direct functionalization of $\mathrm{C}-\mathrm{H}$ bonds of various readily available amino acid derivatives generates a much more straightforward and convenient access to functionalized amino acid derivatives, providing a potential protocol for the direct modification of amino acid derivatives. Despite the remarkable achievements made in the $\mathrm{C}-\mathrm{H}$ functionalization of amino acid derivatives in recent years, ${ }^{6}$ reports on the direct olefination of phenylalanine derivatives at the ortho-position are still rare, and only one kind of directing group, namely sulfonyl amide, was employed successfully to achieve the ortho-C-H olefination of phenylalanine derivatives (Scheme 1a). ${ }^{7}$ Although pioneering works reported by Yu's group in 2008 realized the ortho-alkenylation of

${ }^{a}$ Antibiotics Research and Re-evaluation Key Laboratory of Sichuan Province, Sichuan Industrial Institute of Antibiotics, Chengdu University, 168 Hua Guan Road, Chengdu 610052, P. R. China.E-mail: zhaofei@cdu.edu.cn

${ }^{b}$ College of Life Sciences, China Jiliang University, Hangzhou 310018, Zhejiang, P. R. China

$\uparrow$ Electronic supplementary information (ESI) available: Experimental details and additional spectra. See DOI: 10.1039/c7ra02574b phenylalanine derivatives assisted by a trifluoro-sulfonamide group, this reaction suffered from the disadvantages of moderate yields, long reaction time and limited examples. ${ }^{7 a}$ Very recently, the ortho-olefination of phenylalanine derivatives was also developed using $N$-methyl- $N$-(2-pyridyl)sulfonyl as the directing group by Carretero's group, but only limited to electrondeficient alkenes. ${ }^{7 b}$ It is noteworthy that both reported methods employed sulfonyl amide as the directing group. In the present work, we report the palladium-catalyzed ortho-olefination of phenylalanine derivatives directed by removable picolinamide group with various electron-deficient and electron-rich alkenes in moderate to high yields (Scheme 1b), thus generating a practical strategy for the direct modification of phenylalanine derivatives to produce olefinated phenylalanine derivatives as potential building blocks for drug discovery. Remarkably, our approach was also compatible with phenylethylamine derivatives, which further broadens the substrate scope and highlights the synthetic utility of this methodology. Our method provides an alternative strategy and additional complementarity to the preexisting methods for the ortho-olefination of phenylalanine and phenylethylamine derivatives.

(a) Previous work:

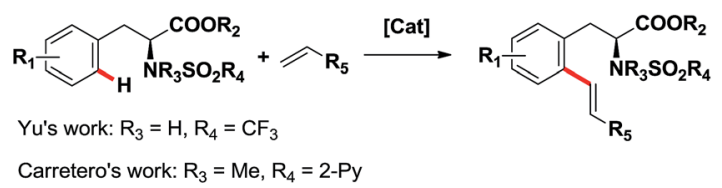

(b) This work:

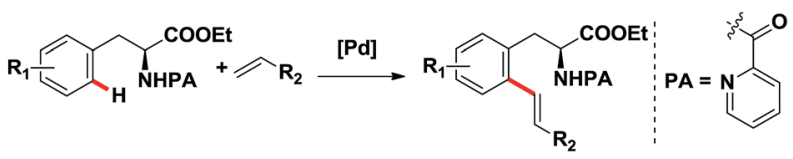

Scheme 1 ortho- $\mathrm{C}-\mathrm{H}$ Olefination of phenylalanine derivatives directed by sulfonyl amide and picolinamide groups. 


\section{Results and discussion}

Initial screening experiments were performed employing 1a and 2a as the model substrates to optimize the reaction conditions (Table 1). We tested the reaction of $1 \mathbf{a}$ and $2 \mathbf{a}$ with $\mathrm{Pd}(\mathrm{OAc})_{2}$ as catalyst in 1,4-dioxane at $110{ }^{\circ} \mathrm{C}$ for $20 \mathrm{~h}$ under an atmosphere of air at first, and the desired product 3a was obtained only in $4 \%$ yield with the starting materials recovered (entry 1 ). Pleasingly, 3a was achieved with a moderate yield (42\%) when oxygen was employed as the oxidant (entry 2). It is worth noting that the addition of $\mathrm{KHCO}_{3}$ as base can significantly improve the yield in the presence of oxidant (entry 3), this might be because $\mathrm{KHCO}_{3}$ can accelerate the deprotonation process to promote this reaction. Subsequently, several common oxidants such as $\mathrm{CuCl}_{2}$, $\mathrm{Cu}(\mathrm{OAc})_{2}, \mathrm{PhI}(\mathrm{OAc})_{2}, \mathrm{~K}_{2} \mathrm{~S}_{2} \mathrm{O}_{8}$, DDQ and BQ were screened, however, none of them gave better yield than oxygen (entries 49). A further screening of the oxidants revealed that employment of silver salts such as $\mathrm{AgNO}_{3}, \mathrm{AgSO}_{3} \mathrm{CF}_{3}, \mathrm{AgOAc}, \mathrm{AgCO}_{2}$ $\mathrm{CF}_{3}, \mathrm{Ag}_{2} \mathrm{O}$ and $\mathrm{Ag}_{2} \mathrm{CO}_{3}$ generally can afford $3 \mathbf{a}$ in moderate to high yields (entries 10-15), and four of them (AgOAc, $\mathrm{AgCO}_{2} \mathrm{CF}_{3}$, $\mathrm{Ag}_{2} \mathrm{O}$ and $\mathrm{Ag}_{2} \mathrm{CO}_{3}$ ) gave higher yield than oxygen (entries 12-15). Among the silver salts screened, $\mathrm{Ag}_{2} \mathrm{CO}_{3}$ was found to be the most efficient oxidant for this transformation with $89 \%$ yield (entry 15). In addition, an exploration of the bases discovered that $\mathrm{KHCO}_{3}$ was the most suitable base for this reaction (entries 15-19). Besides, control experiments showed that $\mathrm{Pd}(\mathrm{OAc})_{2}$

Table 1 Optimization of the reaction conditions ${ }^{a}$

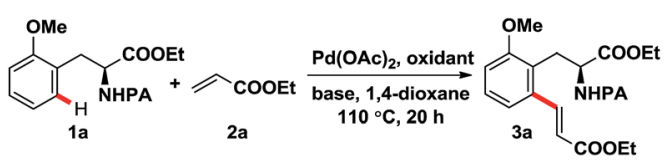

\begin{tabular}{|c|c|c|c|c|}
\hline Entry & Catalyst & Oxidant & Base & Yield $^{b}(\%)$ \\
\hline 1 & $\mathrm{Pd}(\mathrm{OAc})_{2}$ & - & - & 4 \\
\hline 2 & $\mathrm{Pd}(\mathrm{OAc})_{2}$ & 1 atm $\mathrm{O}_{2}$ & - & 42 \\
\hline 3 & $\mathrm{Pd}(\mathrm{OAc})_{2}$ & 1 atm $\mathrm{O}_{2}$ & $\mathrm{KHCO}_{3}$ & 68 \\
\hline 4 & $\mathrm{Pd}(\mathrm{OAc})_{2}$ & $\mathrm{CuCl}_{2}$ & $\mathrm{KHCO}_{3}$ & 7 \\
\hline 5 & $\mathrm{Pd}(\mathrm{OAc})_{2}$ & $\mathrm{Cu}(\mathrm{OAc})_{2}$ & $\mathrm{KHCO}_{3}$ & 36 \\
\hline 6 & $\mathrm{Pd}(\mathrm{OAc})_{2}$ & $\mathrm{PhI}(\mathrm{OAc})_{2}$ & $\mathrm{KHCO}_{3}$ & Trace \\
\hline 7 & $\mathrm{Pd}(\mathrm{OAc})_{2}$ & $\mathrm{~K}_{2} \mathrm{~S}_{2} \mathrm{O}_{8}$ & $\mathrm{KHCO}_{3}$ & 32 \\
\hline 8 & $\mathrm{Pd}(\mathrm{OAc})_{2}$ & DDQ & $\mathrm{KHCO}_{3}$ & Trace \\
\hline 9 & $\mathrm{Pd}(\mathrm{OAc})_{2}$ & BQ & $\mathrm{KHCO}_{3}$ & 67 \\
\hline 10 & $\mathrm{Pd}(\mathrm{OAc})_{2}$ & $\mathrm{AgNO}_{3}$ & $\mathrm{KHCO}_{3}$ & 41 \\
\hline 11 & $\mathrm{Pd}(\mathrm{OAc})_{2}$ & $\mathrm{AgSO}_{3} \mathrm{CF}_{3}$ & $\mathrm{KHCO}_{3}$ & 54 \\
\hline 12 & $\mathrm{Pd}(\mathrm{OAc})_{2}$ & AgOAc & $\mathrm{KHCO}_{3}$ & 72 \\
\hline 13 & $\mathrm{Pd}(\mathrm{OAc})_{2}$ & $\mathrm{AgCO}_{2} \mathrm{CF}_{3}$ & $\mathrm{KHCO}_{3}$ & 81 \\
\hline 14 & $\mathrm{Pd}(\mathrm{OAc})_{2}$ & $\mathrm{Ag}_{2} \mathrm{O}$ & $\mathrm{KHCO}_{3}$ & 83 \\
\hline 15 & $\mathrm{Pd}(\mathrm{OAc})_{2}$ & $\mathrm{Ag}_{2} \mathrm{CO}_{3}$ & $\mathrm{KHCO}_{3}$ & 89 \\
\hline 16 & $\mathrm{Pd}(\mathrm{OAc})_{2}$ & $\mathrm{Ag}_{2} \mathrm{CO}_{3}$ & $\mathrm{~K}_{3} \mathrm{PO}_{4}$ & 78 \\
\hline 17 & $\mathrm{Pd}(\mathrm{OAc})_{2}$ & $\mathrm{Ag}_{2} \mathrm{CO}_{3}$ & $\mathrm{NaHCO}_{3}$ & 80 \\
\hline 18 & $\mathrm{Pd}(\mathrm{OAc})_{2}$ & $\mathrm{Ag}_{2} \mathrm{CO}_{3}$ & $\mathrm{Na}_{2} \mathrm{CO}_{3}$ & 82 \\
\hline 19 & $\mathrm{Pd}(\mathrm{OAc})_{2}$ & $\mathrm{Ag}_{2} \mathrm{CO}_{3}$ & $\mathrm{~K}_{2} \mathrm{CO}_{3}$ & 76 \\
\hline 20 & - & $\mathrm{Ag}_{2} \mathrm{CO}_{3}$ & $\mathrm{KHCO}_{3}$ & $\mathrm{NR}^{c}$ \\
\hline
\end{tabular}

${ }^{a}$ Reaction conditions: 1a ( $\left.0.3 \mathrm{mmol}\right), 2 \mathrm{a}(0.6 \mathrm{mmol}, 2$ equiv. $), \mathrm{Pd}(\mathrm{OAc})_{2}$ (0.03 mmol, $10 \mathrm{~mol} \%$ ), oxidant $(0.6 \mathrm{mmol}, 2$ equiv.), and base (0.6 mmol, 2 equiv.) in 1,4-dioxane $(2.0 \mathrm{ml})$ at $110^{\circ} \mathrm{C}$ for $20 \mathrm{~h}$ in a $25 \mathrm{ml}$ sealed tube. ${ }^{b}$ Isolated yield. ${ }^{c}$ No reaction.
Table 2 Reaction substrate scope of phenylalanine derivatives ${ }^{a}$
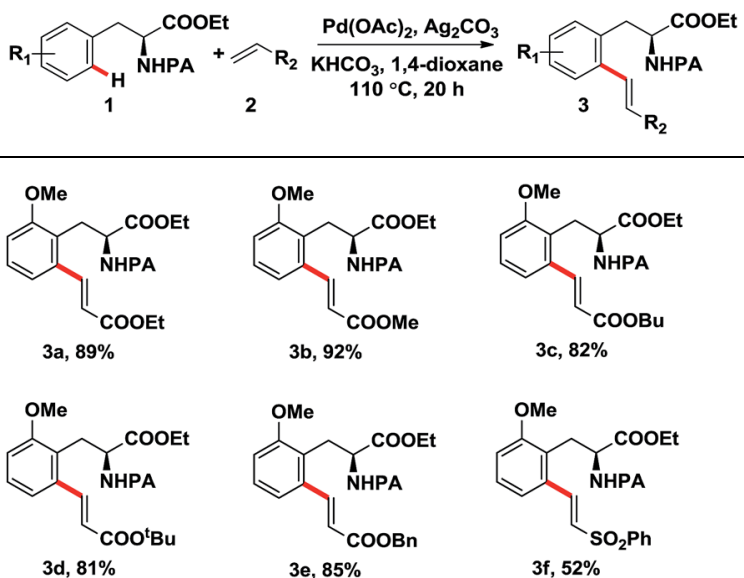

$3 d, 81 \%$

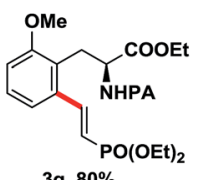

$3 e, 85 \%$

$3 f, 52 \%$
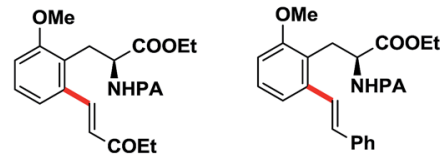

$3 \mathrm{~g}, \mathbf{8 0} \%$

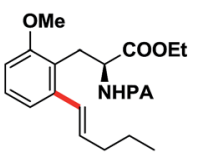

3h, $84 \%$

$3 i, 49 \%^{b}$
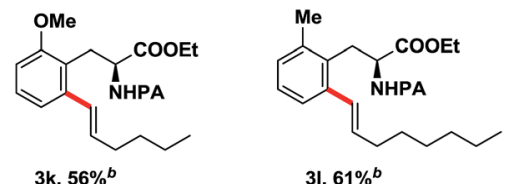

$3 j, 53 \%^{b}$

$3 k, 56 \%^{b}$

$31,61 \%{ }^{b}$
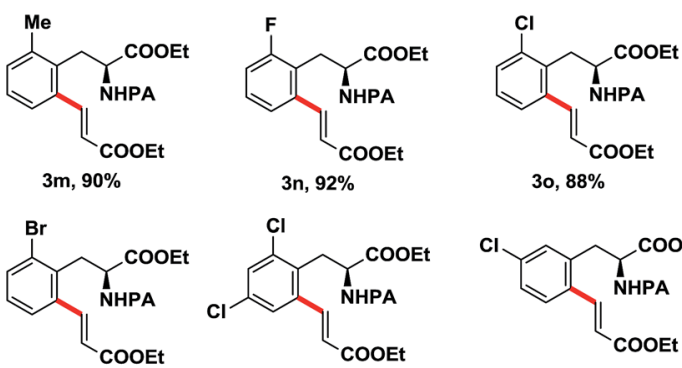

$3 p, 75 \%$

$3 q, 70 \%$

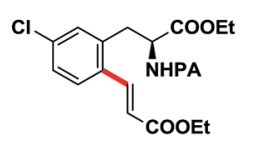

$3 r, 78 \%$
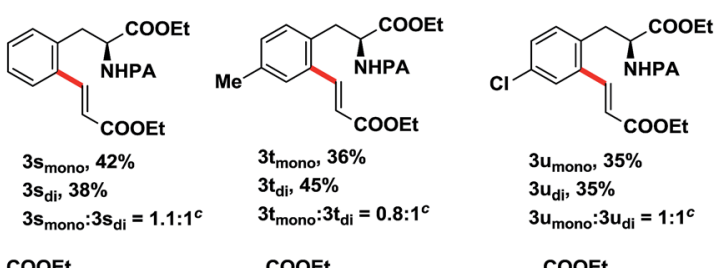

$3 u_{\text {mono }}, 35 \%$

$3 \mathrm{u}_{\mathrm{di}}, 35 \%$

$3 u_{\text {mono }}: 3 u_{d i}=1: 1^{c}$
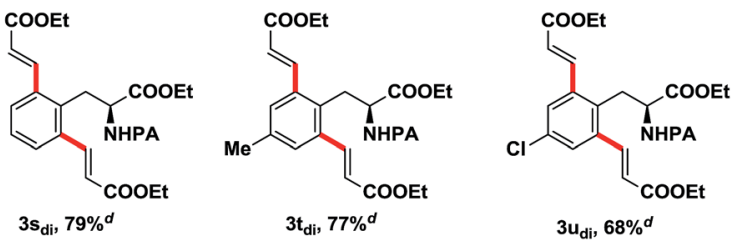

${ }^{a}$ Reaction conditions: 1 (0.3 mmol), 2 (0.6 mmol, 2 equiv.), $\mathrm{Pd}(\mathrm{OAc})_{2}$ (0.03 mmol, $10 \mathrm{~mol} \%$ ), $\mathrm{Ag}_{2} \mathrm{CO}_{3}$ (0.6 mmol, 2 equiv.), and $\mathrm{KHCO}_{3}$ (0.6 mmol, 2 equiv.) in 1,4-dioxane $(2.0 \mathrm{ml})$ at $110{ }^{\circ} \mathrm{C}$ for $20 \mathrm{~h}$ in a $25 \mathrm{ml}$ sealed tube; isolated yields. ${ }^{b} 2$ (1.2 mmol, 4 equiv.), $48 \mathrm{~h}$. ${ }^{c}$ The ratio of mono- and diolefination products was determined by ${ }^{1}$ HNMR. ${ }^{d}$ 2a (0.9 mmol, 3 equiv.), Pd(OAc) $)_{2}$ (0.045 mmol, $\left.15 \mathrm{~mol} \%\right)$, $\mathrm{Ag}_{2} \mathrm{CO}_{3}$ (0.9 mmol, 3 equiv.), and $\mathrm{KHCO}_{3}$ (0.9 mmol, 3 equiv.). 
played a crucial and indispensable role in this ortho-C-H olefination reaction (entry 20). In this way, the optimal reaction conditions were identified using the catalytic system of $\mathrm{Pd}(\mathrm{OAc})_{2} / \mathrm{Ag}_{2} \mathrm{CO}_{3} / \mathrm{KHCO}_{3}$ in 1,4-dioxane at $110{ }^{\circ} \mathrm{C}$ for $20 \mathrm{~h}$.

After determining the optimal reaction conditions, we then examined the general applicability of the process. In general, the olefination process tolerated a variety of phenylalanine derivatives with various substituents at $R_{1}$ and alkenes with electron-donating or electron-withdrawing groups at $\mathrm{R}_{2}$, and furnished the desired products in moderate to high yields (Table 2). The reactions of substrate 1a bearing a methoxy group in the ortho-position with different acrylates afforded the alkenylated products in high yields (3a-3e). Except for acrylates, other activated alkenes, such as vinyl sulfone (3f), vinyl phosphate (3g) and vinyl ketone (3h), could undergo this transformation with moderate to high yields. Notably, unactivated alkenes such as styrene (3i), 1-pentene (3j), 1-hexene (3k) and 1octene (31) were also suitable for this reaction, furnishing the corresponding products in moderate yields. Subsequently, the scope of phenylalanine derivatives was explored. Substrates carrying a substituent ( $\mathrm{Me}, \mathrm{F}, \mathrm{Cl}, \mathrm{Br}$ ) in the ortho-position

Table 3 Reaction substrate scope of phenylethylamine derivatives ${ }^{a}$

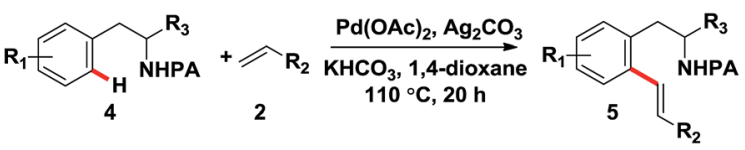

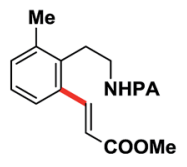

$5 a, 89 \%$

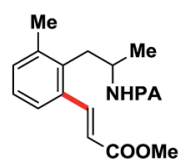

$5 d, 90 \%$

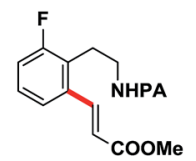

$5 \mathrm{~g}, 82 \%$

$5 \mathrm{i}_{\mathrm{di}}, 75 \%$ c

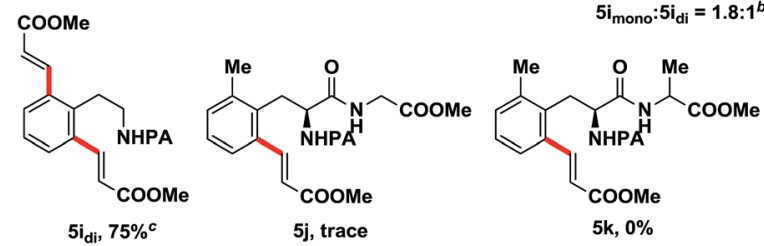

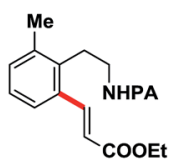

$5 b, 86 \%$

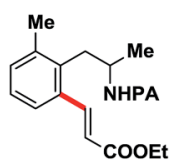

$5 e, 88 \%$

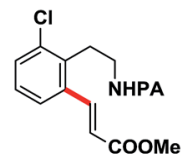
5 h, $79 \%$

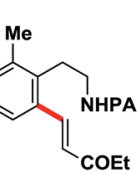

5c, $93 \%$

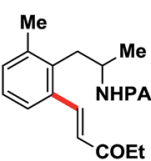

$5 f, 91 \%$

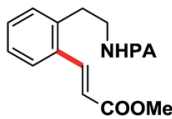

$5 i_{\text {mono }}, 54 \%$

$5 i_{d i}, 30 \%$

$5 i_{m o n o}: 5 i_{d i}=1.8: 1^{b}$

${ }^{a}$ Reaction conditions: 4 ( $\left.0.3 \mathrm{mmol}\right), 2$ (0.6 mmol, 2 equiv.), $\mathrm{Pd}(\mathrm{OAc})_{2}$ (0.03 mmol, $10 \mathrm{~mol} \%), \mathrm{Ag}_{2} \mathrm{CO}_{3}$ (0.6 mmol, 2 equiv.), and $\mathrm{KHCO}_{3}$ (0.6 mmol, 2 equiv.) in 1,4-dioxane $(2.0 \mathrm{ml})$ at $110^{\circ} \mathrm{C}$ for $20 \mathrm{~h}$ in a $25 \mathrm{ml}$ sealed tube; isolated yields. ${ }^{b}$ The ratio of mono- and diolefination products was determined by ${ }^{1} \mathrm{HNMR} .{ }^{c}$ Methyl acrylate (0.9 mmol, 3 equiv.), $\mathrm{Pd}(\mathrm{OAc})_{2}$ (0.045 mmol, $\left.15 \mathrm{~mol} \%\right), \mathrm{Ag}_{2} \mathrm{CO}_{3}$ (0.9 mmol, 3 equiv.), and $\mathrm{KHCO}_{3}(0.9 \mathrm{mmol}, 3$ equiv.).

reacted smoothly and produced the corresponding products in good to high yields (3m-3q). When meta-substituted substrate was employed, the $\mathrm{C}-\mathrm{H}$ olefination reaction took place at the less sterically hindered position with good yield (3r). The reactions of unsubstituted or para-substituted phenylalanine derivatives afforded a separable mixture of mono- and diolefination products, and exclusive diolefination products were obtained in good yields by increasing the amounts of catalyst, alkene, oxidant and base $(3 \mathbf{s}-\mathbf{3 u})$, this may attribute to the transformation of monoolefination products which were produced in the reaction process into diolefination products when excessive reagents were used.

To further broaden the substrate scope of this process, phenylethylamine derivatives were tested as the reaction substrates. To our delight, this approach was also compatible with phenylethylamines (Table 3). The reactions of diverse substituted phenylethylamine derivatives with various alkenes proceeded smoothly under the optimal reaction conditions, affording the desired products in moderate to high yields (5a5i). We also made our efforts to achieve the ortho-C-H olefination of peptides $(\mathbf{5 j} \mathbf{- 5} \mathbf{k})$, albeit with unsuccessful results, this may attribute to the additional coordination of amide of the peptide bond with $\mathrm{Pd}(\mathrm{OAc})_{2}$ which inactivated the catalyst.

To further illustrate the practicality of this methodology, the $\mathrm{C}-\mathrm{H}$ olefination reaction with $\mathbf{1 a}$ was carried out on a gram scale under optimal conditions. Impressively, the desired product $\mathbf{3 a}$ was achieved in $86 \%$ yield (Scheme $2 \mathrm{a}$ ). In addition, the picolinamide directing group can be easily removed using the general and mature protocol reported by Chen (Scheme $2 \mathrm{~b}$ ). ${ }^{\mathbf{8}}$ Besides, removal of the directing group of $\mathbf{5 c}$ and subsequent
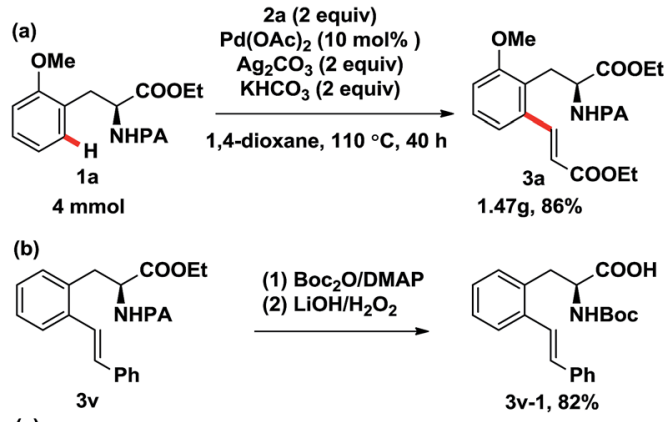

(c)

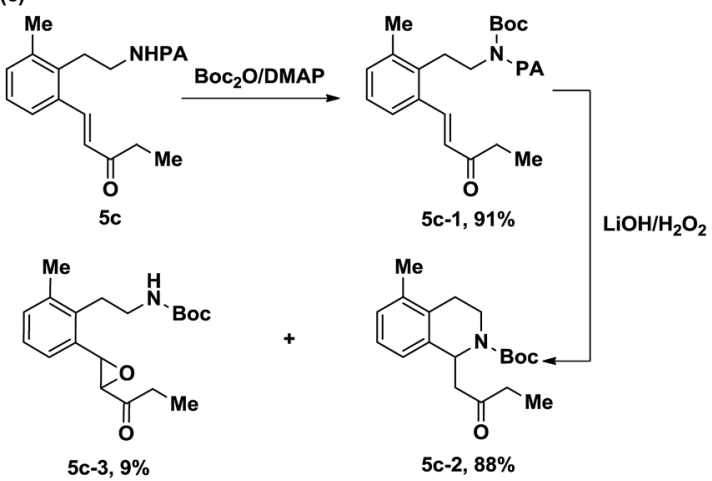

Scheme 2 Gram-scale olefination, removal of the directing group and derivatization. 


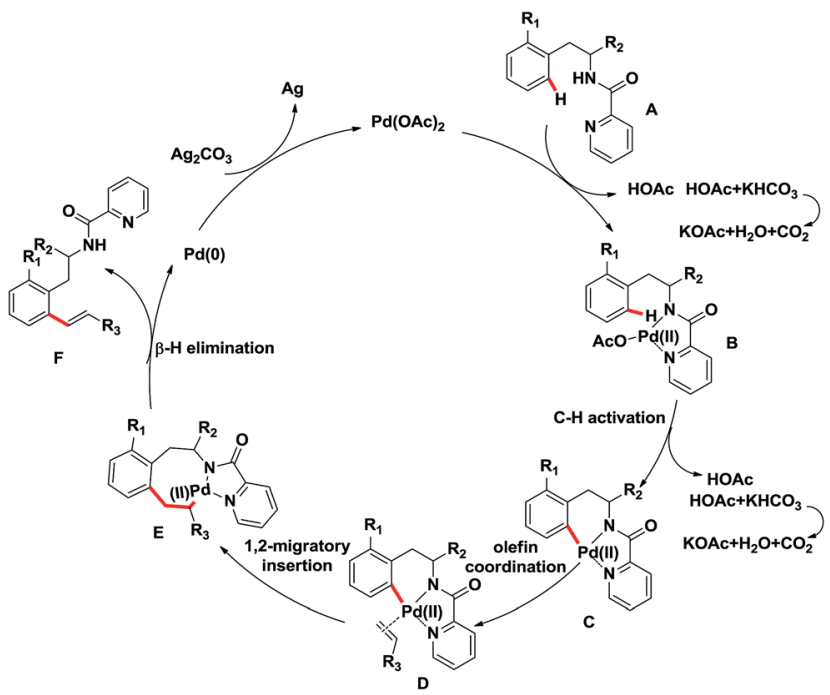

Scheme 3 Proposed reaction mechanism of this ortho- $\mathrm{C}-\mathrm{H}$ olefination of phenylalanine and phenylethylamine derivatives.

intramolecular cyclization gave the tetrahydroisoquinoine $\mathbf{5 c - 2}$ in $80 \%$ yield over two steps, interestingly, epoxide $\mathbf{5 c - 3}$ was obtained as the byproduct in extremely low yield (Scheme 2c). These aspects further highlight the advantages and potential application of this approach.

The plausible mechanism of this ortho-C-H olefination reaction was outlined in Scheme 3. Coordination of amides A to $\mathrm{Pd}(\mathrm{OAc})_{2}$ generates $\mathrm{Pd}(\mathrm{II})$ complex $\mathrm{B}$, subsequent $\mathrm{C}-\mathrm{H}$ bond activation at the ortho-position forms the $\mathrm{Ar}-\mathrm{Pd}$ metastable intermediate $\mathrm{C}$. Then, olefin coordination and 1,2-migratory insertion take place sequentially followed by $\beta-\mathrm{H}$ elimination, affording the olefination products $\mathrm{F}$ and a $\operatorname{Pd}(0)$ species. The reduced $\mathrm{Pd}(0)$ species was oxidized to $\mathrm{Pd}(\mathrm{II})$ oxidation state by $\mathrm{Ag}_{2} \mathrm{CO}_{3}$ to regenerate the $\mathrm{Pd}(\mathrm{II})$ catalyst. $\mathrm{KHCO}_{3}$ may play as a base which can accelerate the deprotonation process to promote this reaction.

\section{Conclusions}

In conclusion, we have developed an efficient and practical approach for the ortho-C-H olefination of phenylalanine and phenylethylamine derivatives with the assistance of removable picolinamide group. The broad substrate scope, functional tolerance, and moderate to high yields demonstrate the great potential of this method for the direct modification of phenylalanine and phenylethylamine derivatives. Other synthetic applications of this method are currently under investigation in our laboratory.

\section{Experimental section}

\section{General information}

The reagents were purchased from commercial suppliers and used without further purification. Analytical thin-layer chromatography (TLC) was performed on HSGF 254 (0.15-0.2 mm thickness), visualized by irradiation with UV light $(254 \mathrm{~nm})$.
Column chromatography was performed using silica gel FCP 200-300. Melting points were measured with a micro melting point apparatus. Nuclear magnetic resonance spectra were recorded on a Brucker AMX-400 or AMX-500 MHz instrument (TMS as IS). Chemical shifts were reported in parts per million $(\mathrm{ppm}, \delta)$ downfield from tetramethylsilane. Proton coupling patterns were described as singlet $(\mathrm{s})$, doublet $(\mathrm{d})$, triplet $(\mathrm{t})$, quartet (q), multiplet (m), and broad (br). Low- and highresolution mass spectra (LRMS and HRMS) were measured on a spectrometer.

\section{Preparation and characterization data of compound 3}

Procedure for the preparation of 3a. A $25 \mathrm{ml}$ Schlenk tube equipped with a magnetic stir bar was charged with $2 \mathrm{a}(98.5 \mathrm{mg}$, $0.3 \mathrm{mmol})$, ethyl acrylate $(65.2 \mu \mathrm{l}, 0.6 \mathrm{mmol}), \mathrm{Pd}(\mathrm{OAc})_{2}(6.7 \mathrm{mg}$, $0.03 \mathrm{mmol}$ ), $\mathrm{Ag}_{2} \mathrm{CO}_{3}(165.5 \mathrm{mg}, 0.6 \mathrm{mmol}), \mathrm{KHCO}_{3}(60.1 \mathrm{mg}, 0.6$ $\mathrm{mmol})$ and 1,4-dioxane $(2.0 \mathrm{ml})$ and then capped with a septa. After that, the tube was kept in the preheated oil bath at $110{ }^{\circ} \mathrm{C}$ for $20 \mathrm{~h}$. After removal of the solvent, the residue was purified by flash chromatography on silica gel to give the desired product 3a as white solid.

Compounds $3 b-3 v$ and $5 a-5 i$ were prepared following the similar procedure carried out for $3 \mathbf{a}$

(S,E)-Ethyl-3-(2-(3-ethoxy-3-oxo-2-(picolinamido)propyl)-3methoxyphenyl)acrylate (3a). White solid (113.9 mg, yield 89\%), mp 125-126 ${ }^{\circ} \mathrm{C} .{ }^{1} \mathrm{H}$ NMR $\left(400 \mathrm{MHz}, \mathrm{CDCl}_{3}\right) \delta 8.83(\mathrm{~d}, J=7.6 \mathrm{~Hz}$, $1 \mathrm{H}), 8.53(\mathrm{~d}, J=4.7 \mathrm{~Hz}, 1 \mathrm{H}), 8.12-8.01(\mathrm{~m}, 2 \mathrm{H}), 7.82-7.73(\mathrm{~m}$, $1 \mathrm{H}), 7.43-7.34(\mathrm{~m}, 1 \mathrm{H}), 7.24-7.18(\mathrm{~m}, 1 \mathrm{H}), 7.18-7.12(\mathrm{~m}, 1 \mathrm{H})$, $6.87(\mathrm{~d}, J=8.1 \mathrm{~Hz}, 1 \mathrm{H}), 6.30(\mathrm{~d}, J=15.7 \mathrm{~Hz}, 1 \mathrm{H}), 4.88-4.75(\mathrm{~m}$, $1 \mathrm{H}), 4.30-4.13(\mathrm{~m}, 4 \mathrm{H}), 3.91(\mathrm{~s}, 3 \mathrm{H}), 3.50-3.33(\mathrm{~m}, 2 \mathrm{H}), 1.34(\mathrm{t}, J$ $=7.1 \mathrm{~Hz}, 3 \mathrm{H}), 1.27(\mathrm{t}, J=7.0 \mathrm{~Hz}, 3 \mathrm{H}) ;{ }^{13} \mathrm{C} \mathrm{NMR}(126 \mathrm{MHz}$, $\left.\mathrm{CDCl}_{3}\right) \delta 171.57,166.60,164.36,157.95,149.57,148.11,141.42$, $137.17,135.56,128.33,126.23,124.95,122.28,121.54,119.31$, 111.29, 61.47, 60.60, 55.75, 53.49, 27.97, 14.41, 14.19; LRMS (ESI): $427[\mathrm{M}+\mathrm{H}]^{+}$; HRMS (ESI) calcd for $\mathrm{C}_{23} \mathrm{H}_{27} \mathrm{~N}_{2} \mathrm{O}_{6}[\mathrm{M}+\mathrm{H}]^{+}$ 427.1869, found: 427.1866 .

(S,E)-Methyl-3-(2-(3-ethoxy-3-oxo-2-(picolinamido)propyl)3-methoxyphenyl)acrylate (3b). White solid (113.8 $\mathrm{mg}$, yield 92\%), mp 82-83 ${ }^{\circ} \mathrm{C} .{ }^{1} \mathrm{H}$ NMR $\left(400 \mathrm{MHz}, \mathrm{CDCl}_{3}\right) \delta 8.79(\mathrm{~d}, J=$ $7.5 \mathrm{~Hz}, 1 \mathrm{H}), 8.49(\mathrm{~d}, J=4.4 \mathrm{~Hz}, 1 \mathrm{H}), 8.10-7.97(\mathrm{~m}, 2 \mathrm{H}), 7.77-7.69$ $(\mathrm{m}, 1 \mathrm{H}), 7.38-7.31(\mathrm{~m}, 1 \mathrm{H}), 7.20-7.14(\mathrm{~m}, 1 \mathrm{H}), 7.13-7.06(\mathrm{~m}$, $1 \mathrm{H}), 6.84(\mathrm{~d}, J=8.0 \mathrm{~Hz}, 1 \mathrm{H}), 6.27$ (d, $J=15.7 \mathrm{~Hz}, 1 \mathrm{H}), 4.86-4.72$ (m, 1H), 4.23-4.08 (m, 2H), 3.87 (s, 3H), $3.76(\mathrm{~s}, 3 \mathrm{H}), 3.46-3.30$ $(\mathrm{m}, 2 \mathrm{H}), 1.24(\mathrm{t}, J=6.9 \mathrm{~Hz}, 3 \mathrm{H}) ;{ }^{13} \mathrm{C} \mathrm{NMR}\left(101 \mathrm{MHz}, \mathrm{CDCl}_{3}\right)$ $\delta 171.47,166.94,164.28,157.89,149.44,148.06,141.66,137.11$, 135.38, 128.29, 126.19, 124.91, 122.19, 120.94, 119.19, 111.32, 61.40, 55.69, 53.40, 51.70, 27.91, 14.08; LRMS (ESI): $413[\mathrm{M}+$ $\mathrm{H}]^{+}$; HRMS (ESI) calcd for $\mathrm{C}_{22} \mathrm{H}_{25} \mathrm{~N}_{2} \mathrm{O}_{6}[\mathrm{M}+\mathrm{H}]^{+}$413.1713, found: 413.1709.

(S,E)-Butyl-3-(2-(3-ethoxy-3-oxo-2-(picolinamido)propyl)-3-methoxyphenyl)acrylate (3c). Pale yellow oil (111.8 mg, yield $82 \%) .{ }^{1} \mathrm{H}$ NMR (400 MHz, $\left.\mathrm{CDCl}_{3}\right) \delta 8.81(\mathrm{~d}, J=7.6 \mathrm{~Hz}, 1 \mathrm{H}), 8.50(\mathrm{~d}, J=$ $4.4 \mathrm{~Hz}, 1 \mathrm{H}), 8.11-7.98(\mathrm{~m}, 2 \mathrm{H}), 7.79-7.68(\mathrm{~m}, 1 \mathrm{H}), 7.41-7.30(\mathrm{~m}$, 1H), 7.20-7.08 (m, 2H), $6.84(\mathrm{~d}, J=7.9 \mathrm{~Hz}, 1 \mathrm{H}), 6.28(\mathrm{~d}, J=$ $15.7 \mathrm{~Hz}, 1 \mathrm{H}), 4.84-4.73(\mathrm{~m}, 1 \mathrm{H}), 4.23-4.09(\mathrm{~m}, 4 \mathrm{H}), 3.88(\mathrm{~s}, 3 \mathrm{H})$, 3.47-3.30 (m, 2H), 1.71-1.62 (m, 2H), 1.47-1.37 (m, 2H), $1.25(\mathrm{t}, J$ 
$=7.0 \mathrm{~Hz}, 3 \mathrm{H}), 0.94(\mathrm{t}, J=7.4 \mathrm{~Hz}, 3 \mathrm{H}) ;{ }^{13} \mathrm{C} \mathrm{NMR}\left(101 \mathrm{MHz}, \mathrm{CDCl}_{3}\right)$ $\delta$ 171.52, 166.66, 164.31, 157.91, 149.50, 148.07, 141.36, 137.13, $135.50,128.28,126.19,124.88,122.22,121.47,119.25,111.25$, 64.49, 61.42, 55.71, 53.43, 30.80, 27.92, 19.25, 14.14, 13.81; LRMS (ESI): $455[\mathrm{M}+\mathrm{H}]^{+}$; HRMS (ESI) calcd for $\mathrm{C}_{25} \mathrm{H}_{31} \mathrm{~N}_{2} \mathrm{O}_{6}[\mathrm{M}+\mathrm{H}]^{+}$ 455.2182, found: 455.2180 .

(S,E)-tert-Butyl-3-(2-(3-ethoxy-3-oxo-2-(picolinamido)propyl)-3methoxyphenyl)acrylate (3d). White solid (110.4 mg, yield 81\%), mp 80-81 ${ }^{\circ} \mathrm{C} .{ }^{1} \mathrm{H}$ NMR $\left(400 \mathrm{MHz}, \mathrm{CDCl}_{3}\right) \delta 8.85$ (d, $J=7.3 \mathrm{~Hz}$, $1 \mathrm{H}), 8.53(\mathrm{~d}, J=4.2 \mathrm{~Hz}, 1 \mathrm{H}), 8.07$ (d, $J=7.7 \mathrm{~Hz}, 1 \mathrm{H}), 7.96$ (d, $J=$ 15.6 Hz, 1H), 7.83-7.72 (m, 1H), 7.43-7.32 (m, 1H), 7.22-7.10 $(\mathrm{m}, 2 \mathrm{H}), 6.85(\mathrm{~d}, J=8.0 \mathrm{~Hz}, 1 \mathrm{H}), 6.24(\mathrm{~d}, J=15.7 \mathrm{~Hz}, 1 \mathrm{H}), 4.87-$ $4.74(\mathrm{~m}, 1 \mathrm{H}), 4.27-4.12(\mathrm{~m}, 2 \mathrm{H}), 3.91(\mathrm{~s}, 3 \mathrm{H}), 3.50-3.30(\mathrm{~m}, 2 \mathrm{H})$, 1.54 (s, 9H), 1.26 (t, $J=7.0 \mathrm{~Hz}, 3 \mathrm{H}) ;{ }^{13} \mathrm{C} \mathrm{NMR}\left(126 \mathrm{MHz} \mathrm{CDCl}_{3}\right)$ $\delta 171.61,165.93,164.40,157.91,149.60,148.11,140.29,137.17$, $135.70,128.25,126.21,124.78,123.41,122.28,119.31,111.06$, 80.58, 61.45, 55.73, 53.53, 28.28, 27.87, 14.21; LRMS (ESI): 455 $[\mathrm{M}+\mathrm{H}]^{+}$; HRMS (ESI) calcd for $\mathrm{C}_{25} \mathrm{H}_{31} \mathrm{~N}_{2} \mathrm{O}_{6}[\mathrm{M}+\mathrm{H}]^{+}$455.2182, found: 455.2178 .

(S,E)-Benzyl-3-(2-(3-ethoxy-3-oxo-2-(picolinamido)propyl)-3-metho xyphenyl)acrylate (3e). Pale yellow oil (124.6 mg, yield $85 \%) .{ }^{1} \mathrm{H}$ NMR (400 MHz, $\left.\mathrm{CDCl}_{3}\right) \delta 8.72(\mathrm{~d}, J=7.6 \mathrm{~Hz}, 1 \mathrm{H}), 8.37(\mathrm{~d}, J=$ $4.2 \mathrm{~Hz}, 1 \mathrm{H}), 8.03$ (d, $J=15.7 \mathrm{~Hz}, 1 \mathrm{H}), 7.95(\mathrm{~d}, J=7.8 \mathrm{~Hz}, 1 \mathrm{H})$, 7.67-7.59 (m, 1H), 7.35-7.30 (m, 2H), 7.29-7.21 (m, 4H), 7.12-7.06 (m, 1H), 7.05-7.01 (m, 1H), $6.76(\mathrm{~d}, J=7.8 \mathrm{~Hz}, 1 \mathrm{H}), 6.24(\mathrm{~d}, J=$ $15.7 \mathrm{~Hz}, 1 \mathrm{H}), 5.15$ (s, 2H), 4.80-4.68 (m, 1H), 4.13-3.97 (m, 2H), 3.79 (s, 3H), 3.38-3.24 (m, 2H), $1.12(\mathrm{t}, J=7.1 \mathrm{~Hz}, 3 \mathrm{H}) ;{ }^{13} \mathrm{C}$ NMR $\left(101 \mathrm{MHz}, \mathrm{CDCl}_{3}\right) \delta 171.51,166.38,164.31,157.95,149.50,148.09$, $142.04,137.13,136.15,135.41,128.64,128.33,128.27,126.20$, 125.02, 122.24, 121.03, 119.28, 111.40, 66.38, 61.44, 55.73, 53.40, 28.01, 14.14; LRMS (ESI): $511[\mathrm{M}+\mathrm{Na}]^{+}$; HRMS (ESI) calcd for $\mathrm{C}_{28} \mathrm{H}_{28} \mathrm{~N}_{2} \mathrm{O}_{6} \mathrm{Na}[\mathrm{M}+\mathrm{Na}]^{+}$511.1845, found: 511.1840 .

(S,E)-Ethyl-3-(2-methoxy-6-(2-(phenylsulfonyl)vinyl)phenyl)-2(picolinamido)propanoate (3f). Pale yellow oil (75.7 mg, yield $52 \%) .{ }^{1} \mathrm{H}$ NMR $\left(400 \mathrm{MHz}, \mathrm{CDCl}_{3}\right) \delta 8.84(\mathrm{~d}, J=7.5 \mathrm{~Hz}, 1 \mathrm{H}), 8.58-$ $8.51(\mathrm{~m}, 1 \mathrm{H}), 8.13-8.03(\mathrm{~m}, 2 \mathrm{H}), 8.03-7.95(\mathrm{~m}, 2 \mathrm{H}), 7.80-7.75$ (m, $1 \mathrm{H}), 7.62-7.52(\mathrm{~m}, 3 \mathrm{H}), 7.39$ (ddd, $J=7.5,4.8,1.2 \mathrm{~Hz}, 1 \mathrm{H})$, $7.23-7.14(\mathrm{~m}, 1 \mathrm{H}), 7.04(\mathrm{~d}, J=7.9 \mathrm{~Hz}, 1 \mathrm{H}), 6.89$ (d, $J=8.2 \mathrm{~Hz}$, $1 \mathrm{H}), 6.78(\mathrm{~d}, J=15.1 \mathrm{~Hz}, 1 \mathrm{H}), 4.80-4.68(\mathrm{~m}, 1 \mathrm{H}), 4.27-4.14(\mathrm{~m}$, 2H), 3.91 (s, 3H), 3.46 (dd, $J=13.9,9.6 \mathrm{~Hz}, 1 \mathrm{H}), 3.35$ (dd, $J=$ 14.0, 5.2 Hz, 1H), 1.27 (t, $J=6.7 \mathrm{~Hz}, 3 \mathrm{H}) ;{ }^{13} \mathrm{C}$ NMR $(126 \mathrm{MHz}$, $\left.\mathrm{CDCl}_{3}\right) \delta 171.47,164.42,158.11,149.44,148.22,140.65,139.73$, 137.26, 133.51, 133.29, 130.44, 129.47, 128.56, 127.95, 126.34, 125.74, 122.30, 119.57, 112.21, 61.68, 55.87, 53.61, 28.25, 14.24; LRMS (ESI): $495[\mathrm{M}+\mathrm{H}]^{+}$; HRMS (ESI) calcd for $\mathrm{C}_{26} \mathrm{H}_{27} \mathrm{~N}_{2} \mathrm{O}_{6} \mathrm{~S}[\mathrm{M}$ $+\mathrm{H}]^{+}$495.1590, found: 495.1592.

(S,E)-Ethyl-3-(2-(2-(diethoxyphosphoryl)vinyl)-6-methoxyphenyl)2-(picolinamido)propanoate (3g). Pale yellow oil (117.7 mg, yield $80 \%) .{ }^{1} \mathrm{H}$ NMR $\left(400 \mathrm{MHz}, \mathrm{CDCl}_{3}\right) \delta 8.87(\mathrm{~d}, J=7.4 \mathrm{~Hz}, 1 \mathrm{H}), 8.58-$ $8.49(\mathrm{~m}, 1 \mathrm{H}), 8.08-8.01(\mathrm{~m}, 1 \mathrm{H}), 7.88-7.73(\mathrm{~m}, 2 \mathrm{H}), 7.37$ (ddd, $J=$ 7.6, 4.8, 1.2 Hz, 1H), 7.24-7.15 (m, 1H), $7.11(\mathrm{~d}, J=7.3 \mathrm{~Hz}, 1 \mathrm{H})$, $6.85(\mathrm{~d}, J=7.8 \mathrm{~Hz}, 1 \mathrm{H}), 6.19(\mathrm{dd}, J=18.7,17.2 \mathrm{~Hz}, 1 \mathrm{H}), 4.82-4.67$ (m, 1H), 4.24-4.08 (m, 6H), $3.91(\mathrm{~s}, 3 \mathrm{H}), 3.43$ (dd, $J=13.9$, $10.0 \mathrm{~Hz}, 1 \mathrm{H}), 3.30(\mathrm{dd}, J=13.9,5.1 \mathrm{~Hz}, 1 \mathrm{H}), 1.36(\mathrm{t}, J=7.1 \mathrm{~Hz}$, $3 \mathrm{H}), 1.35(\mathrm{t}, J=7.1 \mathrm{~Hz}, 3 \mathrm{H}), 1.23(\mathrm{t}, J=7.1 \mathrm{~Hz}, 3 \mathrm{H}) ;{ }^{13} \mathrm{C} \mathrm{NMR}(126$ $\left.\mathrm{MHz}, \mathrm{CDCl}_{3}\right) \delta 171.55,164.35,157.81,149.39,148.08,145.20(\mathrm{~d}$, $\left.J_{\mathrm{C}-\mathrm{P}}=6.8 \mathrm{~Hz}\right), 137.15,135.98\left(\mathrm{~d}, J_{\mathrm{C}-\mathrm{P}}=20.2 \mathrm{~Hz}\right), 128.28,126.22$, $124.39,122.16,119.00,117.90\left(\mathrm{~d}, J_{\mathrm{C}-\mathrm{P}}=189.7 \mathrm{~Hz}\right), 111.21,62.03$ $\left(\mathrm{d}, J_{\mathrm{C}-\mathrm{P}}=6.6 \mathrm{~Hz}\right), 61.98\left(\mathrm{~d}, J_{\mathrm{C}-\mathrm{P}}=6.3 \mathrm{~Hz}\right), 61.40,55.70,53.42$, 27.79, 16.46, 16.41, 14.10; LRMS (ESI): 491 [M + H] $]^{+}$; HRMS (ESI) calcd for $\mathrm{C}_{24} \mathrm{H}_{32} \mathrm{~N}_{2} \mathrm{O}_{7} \mathrm{P}[\mathrm{M}+\mathrm{H}]^{+}$491.1947, found: 491.1941 .

(S,E)-Ethyl-3-(2-methoxy-6-(3-oxopent-1-en-1-yl)phenyl)-2-(picolin amido)propanoate (3h). White solid (103.4 mg, yield $84 \%), \mathrm{mp}$ 81-82 ${ }^{\circ} \mathrm{C} .{ }^{1} \mathrm{H}$ NMR $\left(400 \mathrm{MHz}, \mathrm{CDCl}_{3}\right) \delta 8.83(\mathrm{~d}, J=7.3 \mathrm{~Hz}, 1 \mathrm{H})$, 8.57-8.50 (m, 1H), 8.12-8.06 (m, 1H), 7.96 (d, $J=16.1 \mathrm{~Hz}, 1 \mathrm{H})$, 7.84-7.76 (m, 1H), 7.41 (ddd, $J=7.6,4.8,1.2 \mathrm{~Hz}, 1 \mathrm{H}$ ), 7.25-7.15 (m, 2H), 6.92-6.86 (m, 1H), 6.55 (d, $J=16.1 \mathrm{~Hz}, 1 \mathrm{H}), 4.85-4.72$ $(\mathrm{m}, 1 \mathrm{H}), 4.26-4.11(\mathrm{~m}, 2 \mathrm{H}), 3.91(\mathrm{~s}, 3 \mathrm{H}), 3.48(\mathrm{dd}, J=14.0,9.0 \mathrm{~Hz}$, $1 \mathrm{H}), 3.37$ (dd, $J=14.0,5.4 \mathrm{~Hz}, 1 \mathrm{H}), 2.77$ (q, $J=7.3 \mathrm{~Hz}, 2 \mathrm{H}), 1.24$ $(\mathrm{t}, J=7.2 \mathrm{~Hz}, 3 \mathrm{H}), 1.17(\mathrm{t}, J=7.3 \mathrm{~Hz}, 3 \mathrm{H}) ;{ }^{13} \mathrm{C}$ NMR $(101 \mathrm{MHz}$, $\left.\mathrm{CDCl}_{3}\right) \delta 201.24,171.64,164.38,158.04,149.53,148.18,139.34$, $137.33,135.75,129.76,128.43,126.38,125.17,122.36,119.23$, 111.43, 61.59, 55.81, 53.66, 33.08, 28.16, 14.23, 8.36; LRMS (ESI): $433[\mathrm{M}+\mathrm{Na}]^{+}$; HRMS (ESI) calcd for $\mathrm{C}_{23} \mathrm{H}_{26} \mathrm{~N}_{2} \mathrm{O}_{5} \mathrm{Na}[\mathrm{M}+\mathrm{Na}]^{+}$ 433.1739, found: 433.1738.

(S,E)-Ethyl-3-(2-methoxy-6-styrylphenyl)-2-(picolinamido)propanoate (3i). White solid (62.5 mg, yield 49\%), mp 107-108 ${ }^{\circ} \mathrm{C} .{ }^{1} \mathrm{H}$ NMR $\left(400 \mathrm{MHz}, \mathrm{CDCl}_{3}\right) \delta 8.95(\mathrm{~d}, J=6.7 \mathrm{~Hz}, 1 \mathrm{H}), 8.49(\mathrm{~d}, J=4.7 \mathrm{~Hz}$, $1 \mathrm{H}), 8.06(\mathrm{~d}, J=7.8 \mathrm{~Hz}, 1 \mathrm{H}), 7.78-7.71(\mathrm{~m}, 1 \mathrm{H}), 7.59-7.48(\mathrm{~m}$, $3 \mathrm{H}), 7.41-7.33(\mathrm{~m}, 3 \mathrm{H}), 7.29-7.20(\mathrm{~m}, 3 \mathrm{H}), 6.98(\mathrm{~d}, J=16.0 \mathrm{~Hz}$, $1 \mathrm{H}), 6.80(\mathrm{~d}, J=6.7 \mathrm{~Hz}, 1 \mathrm{H}), 4.86-4.74(\mathrm{~m}, 1 \mathrm{H}), 4.28-4.12(\mathrm{~m}$, $2 \mathrm{H}), 3.93(\mathrm{~s}, 3 \mathrm{H}), 3.50(\mathrm{dd}, J=14.0,9.5 \mathrm{~Hz}, 1 \mathrm{H}), 3.38(\mathrm{dd}, J=$ 14.0, $4.9 \mathrm{~Hz}, 1 \mathrm{H}), 1.28$ (t, $J=7.2 \mathrm{~Hz}, 3 \mathrm{H}) ;{ }^{13} \mathrm{C}$ NMR $(126 \mathrm{MHz}$, $\left.\mathrm{CDCl}_{3}\right) \delta 172.01,164.60,157.85,149.67,148.12,138.43,137.50$, $137.15,131.80,128.78,128.11,127.91,126.87,126.21,125.54$, 123.35, 122.32, 118.59, 109.17, 61.49, 55.70, 53.95, 28.04, 14.22; LRMS (ESI): 453 [M + Na] ${ }^{+}$; HRMS (ESI) calcd for $\mathrm{C}_{26} \mathrm{H}_{26} \mathrm{~N}_{2} \mathrm{O}_{4} \mathrm{Na}$ $[\mathrm{M}+\mathrm{Na}]^{+}$453.1790, found: 453.1786.

(S,E)-Ethyl-3-(2-methoxy-6-(pent-1-en-1-yl)phenyl)-2-(picolinamido) propanoate (3j). Pale yellow oil (63.3 mg, yield 53\%). ${ }^{1} \mathrm{H}$ NMR $\left(400 \mathrm{MHz}, \mathrm{CDCl}_{3}\right) \delta 8.95(\mathrm{~d}, J=6.7 \mathrm{~Hz}, 1 \mathrm{H}), 8.54(\mathrm{~d}, J=4.4 \mathrm{~Hz}$, $1 \mathrm{H}), 8.09(\mathrm{~d}, J=7.8 \mathrm{~Hz}, 1 \mathrm{H}), 7.83-7.73(\mathrm{~m}, 1 \mathrm{H}), 7.39(\mathrm{dd}, J=$ $6.6,4.8 \mathrm{~Hz}, 1 \mathrm{H}), 7.18-7.09(\mathrm{~m}, 1 \mathrm{H}), 7.05(\mathrm{~d}, J=7.6 \mathrm{~Hz}, 1 \mathrm{H})$, 6.78-6.64 (m, 2H), 6.17-6.04 (m, 1H), 4.79-4.65 (m, 1H), 4.27$4.14(\mathrm{~m}, 2 \mathrm{H}), 3.92(\mathrm{~s}, 3 \mathrm{H}), 3.40(\mathrm{dd}, J=13.8,10.1 \mathrm{~Hz}, 1 \mathrm{H}), 3.24$ (dd, $J=13.8,4.8 \mathrm{~Hz}, 1 \mathrm{H}), 2.30-2.10(\mathrm{~m}, 2 \mathrm{H}), 1.55-1.46(\mathrm{~m}, 2 \mathrm{H})$, $1.27(\mathrm{t}, J=7.2 \mathrm{~Hz}, 3 \mathrm{H}), 0.97(\mathrm{t}, J=7.4 \mathrm{~Hz}, 3 \mathrm{H}) ;{ }^{13} \mathrm{C}$ NMR $(101$ $\left.\mathrm{MHz}, \mathrm{CDCl}_{3}\right) \delta 172.17,164.65,157.66,149.78,148.13,139.25$, $137.20,134.57,127.90,126.90,126.22,122.40,122.34,119.03$, 108.44, 61.36, 55.64, 53.82, 35.50, 27.85, 22.50, 14.25, 13.92; LRMS (ESI): $397[\mathrm{M}+\mathrm{H}]^{+}$; HRMS (ESI) calcd for $\mathrm{C}_{23} \mathrm{H}_{29} \mathrm{~N}_{2} \mathrm{O}_{4}[\mathrm{M}$ $+\mathrm{H}]^{+}$397.2127, found: 397.2119 .

(S,E)-Ethyl-3-(2-(hex-1-en-1-yl)-6-methoxyphenyl)-2-(picolinamido)propanoate (3k). Pale yellow oil (69.2 mg, yield 56\%). ${ }^{1} \mathrm{H}$ NMR (400 $\left.\mathrm{MHz}, \mathrm{CDCl}_{3}\right) \delta 8.94(\mathrm{~d}, J=6.7 \mathrm{~Hz}, 1 \mathrm{H}), 8.58-8.47(\mathrm{~m}, 1 \mathrm{H}), 8.09(\mathrm{~d}, J$ $=7.8 \mathrm{~Hz}, 1 \mathrm{H}), 7.82-7.72(\mathrm{~m}, 1 \mathrm{H}), 7.38(\mathrm{ddd}, J=7.6,4.8,1.2 \mathrm{~Hz}$, 1H), 7.18-7.09 (m, 1H), $7.04(\mathrm{~d}, J=7.5 \mathrm{~Hz}, 1 \mathrm{H}), 6.77-6.64(\mathrm{~m}, 2 \mathrm{H})$, 6.18-6.02 (m, 1H), 4.77-4.67 (m, 1H), 4.26-4.15 (m, 2H), $3.92(\mathrm{~s}$, $3 \mathrm{H}), 3.40(\mathrm{dd}, J=13.8,10.2 \mathrm{~Hz}, 1 \mathrm{H}), 3.24(\mathrm{dd}, J=13.8,4.8 \mathrm{~Hz}, 1 \mathrm{H})$, 2.29-2.16 (m, 2H), 1.49-1.35 (m, 4H), $1.26(\mathrm{t}, J=7.2 \mathrm{~Hz}, 3 \mathrm{H}), 0.93$ $(\mathrm{t}, J=7.2 \mathrm{~Hz}, 3 \mathrm{H}) ;{ }^{13} \mathrm{C} \mathrm{NMR}\left(101 \mathrm{MHz}, \mathrm{CDCl}_{3}\right) \delta 172.17,164.64$, $157.67,149.82$, 148.13, 139.27, 137.19, 134.79, 127.89, 126.71, $126.20,122.40,122.34,119.03,108.43,61.34,55.64,53.81,33.12$, 
31.47, 27.86, 22.46, 14.27, 14.11; LRMS (ESI): $411[\mathrm{M}+\mathrm{H}]^{+}$; HRMS (ESI) calcd for $\mathrm{C}_{24} \mathrm{H}_{31} \mathrm{~N}_{2} \mathrm{O}_{4}[\mathrm{M}+\mathrm{H}]^{+}$411.2284, found: 411.2276 .

(S,E)-Ethyl-3-(2-methyl-6-(oct-1-en-1-yl)phenyl)-2-(picolinamido) propanoate (3l). Pale yellow oil (77.3 mg, yield 61\%). ${ }^{1} \mathrm{H}$ NMR $\left(400 \mathrm{MHz}, \mathrm{CDCl}_{3}\right) \delta 8.63-8.50(\mathrm{~m}, 2 \mathrm{H}), 8.11(\mathrm{~d}, J=7.8 \mathrm{~Hz}, 1 \mathrm{H})$, 7.85-7.75 (m, 1H), 7.41 (ddd, $J=7.6,4.8,1.2 \mathrm{~Hz}, 1 \mathrm{H}), 7.24$ (d, $J$ $=7.4 \mathrm{~Hz}, 1 \mathrm{H}), 7.11-6.99(\mathrm{~m}, 2 \mathrm{H}), 6.75(\mathrm{~d}, J=15.5 \mathrm{~Hz}, 1 \mathrm{H}), 6.11-$ $5.97(\mathrm{~m}, 1 \mathrm{H}), 4.99-4.84(\mathrm{~m}, 1 \mathrm{H}), 4.20-3.99(\mathrm{~m}, 2 \mathrm{H}), 3.30(\mathrm{~d}, J=$ $7.9 \mathrm{~Hz}, 2 \mathrm{H}), 2.42(\mathrm{~s}, 3 \mathrm{H}), 2.29-2.16(\mathrm{~m}, 2 \mathrm{H}), 1.52-1.42(\mathrm{~m}, 2 \mathrm{H})$, 1.41-1.29 (m, 6H), $1.13(\mathrm{t}, J=7.1 \mathrm{~Hz}, 3 \mathrm{H}), 0.89(\mathrm{t}, J=6.9 \mathrm{~Hz}$, $3 \mathrm{H}) ;{ }^{13} \mathrm{C}$ NMR $\left(101 \mathrm{MHz}, \mathrm{CDCl}_{3}\right) \delta 172.24,164.12,149.54$, 148.28, 138.57, 137.32, 137.09, 134.46, 132.09, 129.33, 127.86, 127.04, 126.37, 124.63, 122.35, 61.55, 52.68, 33.47, 32.47, 31.90, 29.44, 29.13, 22.79, 20.26, 14.25, 14.02; LRMS (ESI): $423[\mathrm{M}+$ $\mathrm{H}]^{+}$; HRMS (ESI) calcd for $\mathrm{C}_{26} \mathrm{H}_{35} \mathrm{~N}_{2} \mathrm{O}_{3}[\mathrm{M}+\mathrm{H}]^{+}$423.2648, found: 423.2638.

(S,E)-Ethyl-3-(2-(3-ethoxy-3-oxo-2-(picolinamido)propyl)-3methylphenyl)acrylate (3m). White solid (110.8 mg, yield 90\%), mp 61-62 ${ }^{\circ} \mathrm{C} .{ }^{1} \mathrm{H}$ NMR (400 MHz, $\left.\mathrm{CDCl}_{3}\right) \delta 8.57-8.47(\mathrm{~m}, 2 \mathrm{H})$, $8.08(\mathrm{~d}, J=15.7 \mathrm{~Hz}, 1 \mathrm{H}), 8.04(\mathrm{~d}, J=7.8 \mathrm{~Hz}, 1 \mathrm{H}), 7.80-7.71(\mathrm{~m}$, $1 \mathrm{H}), 7.37$ (ddd, $J=7.6,4.8,1.2 \mathrm{~Hz}, 1 \mathrm{H}), 7.32(\mathrm{~d}, J=6.9 \mathrm{~Hz}, 1 \mathrm{H})$, 7.19-7.08 (m, 2H), 6.20 (d, $J=15.6 \mathrm{~Hz}, 1 \mathrm{H}), 4.95-4.84(\mathrm{~m}, 1 \mathrm{H})$, $4.23(\mathrm{q}, J=7.1 \mathrm{~Hz}, 2 \mathrm{H}), 4.16-4.05(\mathrm{~m}, 2 \mathrm{H}), 3.43-3.28(\mathrm{~m}, 2 \mathrm{H})$, $2.44(\mathrm{~s}, 3 \mathrm{H}), 1.31(\mathrm{t}, J=7.1 \mathrm{~Hz}, 3 \mathrm{H}), 1.14(\mathrm{t}, J=7.1 \mathrm{~Hz}, 3 \mathrm{H}) ;{ }^{13} \mathrm{C}$ NMR (101 MHz, $\left.\mathrm{CDCl}_{3}\right) \delta 171.39,166.59,163.85,149.13,148.17$, 142.46, 137.77, 137.16, 134.76, 134.46, 132.19, 127.25, 126.27, 124.88, 122.12, 120.84, 61.59, 60.41, 52.66, 32.36, 20.06, 14.31, 13.91; LRMS (ESI): $411[\mathrm{M}+\mathrm{H}]^{+}$; HRMS (ESI) calcd for $\mathrm{C}_{23} \mathrm{H}_{27} \mathrm{~N}_{2} \mathrm{O}_{5}[\mathrm{M}+\mathrm{H}]^{+}$411.1920, found: 411.1915.

(S,E)-Ethyl-3-(2-(3-ethoxy-3-oxo-2-(picolinamido)propyl)-3-fluorophenyl)acrylate (3n). Pale yellow oil (114.4 mg, yield $92 \%) .{ }^{1} \mathrm{H}$ NMR (400 MHz, $\left.\mathrm{CDCl}_{3}\right) \delta 8.58-8.43(\mathrm{~m}, 2 \mathrm{H}), 8.11-8.03(\mathrm{~m}, 1 \mathrm{H})$, $7.98(\mathrm{~d}, J=15.7 \mathrm{~Hz}, 1 \mathrm{H}), 7.82-7.73(\mathrm{~m}, 1 \mathrm{H}), 7.41-7.35(\mathrm{~m}, 1 \mathrm{H})$, $7.32(\mathrm{~d}, J=7.9 \mathrm{~Hz}, 1 \mathrm{H}), 7.24-7.18(\mathrm{~m}, 1 \mathrm{H}), 7.07-6.98(\mathrm{~m}, 1 \mathrm{H})$, $6.25(\mathrm{~d}, J=15.7 \mathrm{~Hz}, 1 \mathrm{H}), 5.04-4.89(\mathrm{~m}, 1 \mathrm{H}), 4.23-4.12(\mathrm{~m}, 4 \mathrm{H})$, 3.49-3.31 (m, 2H), $1.30(\mathrm{t}, J=7.1 \mathrm{~Hz}, 3 \mathrm{H}), 1.22(\mathrm{t}, J=7.1 \mathrm{~Hz}, 3 \mathrm{H})$; ${ }^{13} \mathrm{C}$ NMR $\left(126 \mathrm{MHz}, \mathrm{CDCl}_{3}\right) \delta 171.06,166.25,164.05,161.82(\mathrm{~d}$, $\left.J_{\mathrm{C}-\mathrm{F}}=245.3 \mathrm{~Hz}\right), 149.25,148.29,140.37\left(\mathrm{~d}, J_{\mathrm{C}-\mathrm{F}}=3.1 \mathrm{~Hz}\right), 137.17$, $136.64\left(\mathrm{~d}, J_{\mathrm{C}-\mathrm{F}}=4.0 \mathrm{~Hz}\right), 128.76\left(\mathrm{~d}, J_{\mathrm{C}-\mathrm{F}}=9.2 \mathrm{~Hz}\right), 126.33,123.42$ $\left(\mathrm{d}, J_{\mathrm{C}-\mathrm{F}}=16.0 \mathrm{~Hz}\right), 122.63\left(\mathrm{~d}, J_{\mathrm{C}-\mathrm{F}}=2.9 \mathrm{~Hz}\right), 122.27,121.95$, $116.36\left(\mathrm{~d}, J_{\mathrm{C}-\mathrm{F}}=23.4 \mathrm{~Hz}\right), 61.82,60.65,52.59,28.22,14.34,14.05$; LRMS (ESI): $415[\mathrm{M}+\mathrm{H}]^{+}$; HRMS (ESI) calcd for $\mathrm{C}_{22} \mathrm{H}_{24} \mathrm{FN}_{2} \mathrm{O}_{5}[\mathrm{M}$ $+\mathrm{H}]^{+}$415.1669, found: 415.1659.

(S,E)-Ethyl-3-(3-chloro-2-(3-ethoxy-3-oxo-2-(picolinamido)propyl)phenyl)acrylate (3o). Pale yellow oil (113.7 mg, yield $88 \%) .{ }^{1} \mathrm{H}$ NMR (400 MHz, $\mathrm{CDCl}_{3}$ ) $\delta 8.57(\mathrm{~d}, J=8.6 \mathrm{~Hz}, 1 \mathrm{H}), 8.54-8.49(\mathrm{~m}$, $1 \mathrm{H})$, 8.11-8.01 (m, 2H), 7.80-7.72 (m, 1H), 7.42-7.33 (m, 3H), $7.20-7.12(\mathrm{~m}, 1 \mathrm{H}), 6.21(\mathrm{~d}, J=15.7 \mathrm{~Hz}, 1 \mathrm{H}), 5.06-4.96(\mathrm{~m}, 1 \mathrm{H})$, 4.26-4.12 (m, 4H), 3.55 (dd, $J=14.1,6.4 \mathrm{~Hz}, 1 \mathrm{H}), 3.47$ (dd, $J=$ $14.1,8.8 \mathrm{~Hz}, 1 \mathrm{H}), 1.32(\mathrm{t}, J=7.1 \mathrm{~Hz}, 3 \mathrm{H}), 1.19(\mathrm{t}, J=7.1 \mathrm{~Hz}, 3 \mathrm{H})$; ${ }^{13} \mathrm{C}$ NMR $\left(101 \mathrm{MHz}, \mathrm{CDCl}_{3}\right) \delta 171.07,166.20,163.97,149.15$, 148.21, 141.20, 137.16, 136.71, 135.95, 133.88, 130.79, 128.40, 126.30, 125.65, 122.35, 122.20, 61.80, 60.66, 52.27, 32.69, 14.31, 14.00; LRMS (ESI): $433\left([\mathrm{M}+\mathrm{H}]^{+},\left(\mathrm{Cl}^{37}\right)\right), 431\left([\mathrm{M}+\mathrm{H}]^{+},\left(\mathrm{Cl}^{35}\right)\right)$; HRMS (ESI) calcd for $\mathrm{C}_{22} \mathrm{H}_{24} \mathrm{ClN}_{2} \mathrm{O}_{5}[\mathrm{M}+\mathrm{H}]^{+} 431.1374$, found: 431.1371 .
(S,E)-Ethyl-3-(3-bromo-2-(3-ethoxy-3-oxo-2-(picolinamido)propyl)phenyl)acrylate (3p). Pale yellow oil $(107.0 \mathrm{mg}$, yield $75 \%) .{ }^{1} \mathrm{H}$ NMR (400 MHz, $\left.\mathrm{CDCl}_{3}\right) \delta 8.59(\mathrm{~d}, J=8.7 \mathrm{~Hz}, 1 \mathrm{H}), 8.52(\mathrm{~d}, J=$ $4.7 \mathrm{~Hz}, 1 \mathrm{H}), 8.11-8.00(\mathrm{~m}, 2 \mathrm{H}), 7.80-7.72(\mathrm{~m}, 1 \mathrm{H}), 7.55$ (dd, $J=$ $7.9,0.9 \mathrm{~Hz}, 1 \mathrm{H}), 7.43-7.35(\mathrm{~m}, 2 \mathrm{H}), 7.12-7.04(\mathrm{~m}, 1 \mathrm{H}), 6.19(\mathrm{~d}, J=$ $15.6 \mathrm{~Hz}, 1 \mathrm{H}), 5.07-4.98$ (m, 1H), 4.28-4.20 (m, 2H), 4.20-4.13 (m, $2 \mathrm{H}), 3.58(\mathrm{dd}, J=14.1,6.3 \mathrm{~Hz}, 1 \mathrm{H}), 3.49(\mathrm{dd}, J=14.1,9.1 \mathrm{~Hz}, 1 \mathrm{H})$, $1.32(\mathrm{t}, J=7.1 \mathrm{~Hz}, 3 \mathrm{H}), 1.19(\mathrm{t}, J=7.1 \mathrm{~Hz}, 3 \mathrm{H}) ;{ }^{13} \mathrm{C}$ NMR (101 $\left.\mathrm{MHz}, \mathrm{CDCl}_{3}\right) \delta 171.10,166.22,164.03,149.27,148.27,141.54$, $137.20,136.91,135.52,134.31,128.78,126.85,126.43,126.32$, 122.55, 122.27, 61.86, 60.73, 52.38, 35.49, 14.38, 14.07; LRMS (ESI): $477\left([\mathrm{M}+\mathrm{H}]^{+},\left(\mathrm{Br}^{81}\right)\right), 475\left([\mathrm{M}+\mathrm{H}]^{+},\left(\mathrm{Br}^{79}\right)\right)$; HRMS (ESI) calcd for $\mathrm{C}_{22} \mathrm{H}_{24} \mathrm{BrN}_{2} \mathrm{O}_{5}[\mathrm{M}+\mathrm{H}]^{+}$475.0869, found: 475.0862 .

(S,E)-Ethyl-3-(3,5-dichloro-2-(3-ethoxy-3-oxo-2-(picolinamido) propyl)phenyl)acrylate (3q). Pale yellow oil (98.1 mg, yield 70\%). ${ }^{1} \mathrm{H}$ NMR (400 MHz, $\mathrm{CDCl}_{3}$ ) $\delta 8.61-8.51(\mathrm{~m}, 2 \mathrm{H}), 8.06(\mathrm{~d}, J=$ $7.8 \mathrm{~Hz}, 1 \mathrm{H}), 8.00$ (d, $J=15.7 \mathrm{~Hz}, 1 \mathrm{H}), 7.84-7.75(\mathrm{~m}, 1 \mathrm{H}), 7.44-$ $7.35(\mathrm{~m}, 3 \mathrm{H}), 6.22$ (d, $J=15.6 \mathrm{~Hz}, 1 \mathrm{H}), 5.06-4.96(\mathrm{~m}, 1 \mathrm{H}), 4.29-$ $4.16(\mathrm{~m}, 4 \mathrm{H}), 3.52$ (dd, $J=14.1,6.2 \mathrm{~Hz}, 1 \mathrm{H}), 3.43(\mathrm{dd}, J=14.1$, $8.8 \mathrm{~Hz}, 1 \mathrm{H}), 1.34(\mathrm{t}, J=7.1 \mathrm{~Hz}, 3 \mathrm{H}), 1.23(\mathrm{t}, J=7.1 \mathrm{~Hz}, 3 \mathrm{H}) ;{ }^{13} \mathrm{C}$ NMR (101 MHz, $\left.\mathrm{CDCl}_{3}\right) \delta 170.98,165.93,164.07,149.17,148.33$, 140.12 , 137.95, 137.33, 136.72, 133.73, 132.61, 130.38, 126.45, 125.84, 123.58, 122.37, 62.05, 60.93, 52.14, 32.60, 14.39, 14.15; LRMS(ESI): $465[\mathrm{M}+\mathrm{H}]^{+}$; HRMS (ESI) calcd for $\mathrm{C}_{22} \mathrm{H}_{23} \mathrm{Cl}_{2} \mathrm{~N}_{2} \mathrm{O}_{5}$ $[\mathrm{M}+\mathrm{H}]^{+}$465.0984, found: 465.0981 .

(S,E)-Ethyl-3-(4-chloro-2-(3-ethoxy-3-oxo-2-(picolinamido)propyl)phenyl)acrylate (3r). White solid (100.8 mg, yield 78\%), mp 84$85{ }^{\circ} \mathrm{C} .{ }^{1} \mathrm{H}$ NMR $\left(400 \mathrm{MHz}, \mathrm{CDCl}_{3}\right) \delta 8.52-8.48(\mathrm{~m}, 1 \mathrm{H}), 8.46(\mathrm{~d}, J=$ $8.4 \mathrm{~Hz}, 1 \mathrm{H}), 8.11-8.06(\mathrm{~m}, 1 \mathrm{H}), 7.90$ (d, $J=15.8 \mathrm{~Hz}, 1 \mathrm{H}), 7.82-7.74$ (m, 1H), 7.49-7.42 (m, 1H), 7.38 (ddd, $J=7.6,4.8,1.2 \mathrm{~Hz}, 1 \mathrm{H}$ ), 7.23-7.16 (m, 2H), 6.22 (d, $J=15.7 \mathrm{~Hz}, 1 \mathrm{H}), 5.05-4.93(\mathrm{~m}, 1 \mathrm{H})$, 4.21-4.07 (m, 4H), 3.37 (dd, $J=14.1,6.2 \mathrm{~Hz}, 1 \mathrm{H}), 3.28(\mathrm{dd}, J=$ 14.1, $6.5 \mathrm{~Hz}, 1 \mathrm{H}), 1.26$ (t, $J=7.1 \mathrm{~Hz}, 3 \mathrm{H}), 1.21$ (t, $J=7.1 \mathrm{~Hz}, 3 \mathrm{H})$; ${ }^{13} \mathrm{C}$ NMR $\left(101 \mathrm{MHz}, \mathrm{CDCl}_{3}\right) \delta 170.75,166.22,163.93,149.05$, $148.24,140.18,137.65,137.16,135.63,132.67,130.95,128.16$, $127.87,126.35,122.22,120.78,61.80,60.48,53.26,35.23,14.23$, 14.04; LRMS (ESI): $433\left([\mathrm{M}+\mathrm{H}]^{+},\left(\mathrm{Cl}^{37}\right)\right), 431\left([\mathrm{M}+\mathrm{H}]^{+},\left(\mathrm{Cl}^{35}\right)\right)$; HRMS (ESI) calcd for $\mathrm{C}_{22} \mathrm{H}_{24} \mathrm{ClN}_{2} \mathrm{O}_{5}[\mathrm{M}+\mathrm{H}]^{+} 431.1374$, found: 431.1369 .

(S,E)-Ethyl-3-(2-(3-ethoxy-3-oxo-2-(picolinamido)propyl)phenyl) acrylate ( $\left.3 \boldsymbol{s}_{\text {mono }}\right)$. White solid (50.0 $\mathrm{mg}$, yield $\left.42 \%\right)$, mp 69$70{ }^{\circ} \mathrm{C} .{ }^{1} \mathrm{H}$ NMR $\left(400 \mathrm{MHz}, \mathrm{CDCl}_{3}\right) \delta 8.55-8.49(\mathrm{~m}, 1 \mathrm{H}), 8.46(\mathrm{~d}, J$ $=8.4 \mathrm{~Hz}, 1 \mathrm{H}), 8.15-8.08(\mathrm{~m}, 1 \mathrm{H}), 8.01(\mathrm{~d}, J=15.7 \mathrm{~Hz}, 1 \mathrm{H}), 7.84-$ $7.76(\mathrm{~m}, 1 \mathrm{H}), 7.58-7.52(\mathrm{~m}, 1 \mathrm{H}), 7.40(\mathrm{ddd}, J=7.5,4.8,1.1 \mathrm{~Hz}$, $1 \mathrm{H}), 7.29-7.22(\mathrm{~m}, 3 \mathrm{H}), 6.28(\mathrm{~d}, J=15.7 \mathrm{~Hz}, 1 \mathrm{H}), 5.06-4.98(\mathrm{~m}$, $1 \mathrm{H}), 4.22-4.10(\mathrm{~m}, 4 \mathrm{H}), 3.47-3.30(\mathrm{~m}, 2 \mathrm{H}), 1.31(\mathrm{t}, J=7.2 \mathrm{~Hz}$, $3 \mathrm{H}), 1.21$ (t, $J=7.1 \mathrm{~Hz}, 3 \mathrm{H}) ;{ }^{13} \mathrm{C} \mathrm{NMR}\left(126 \mathrm{MHz}, \mathrm{CDCl}_{3}\right.$ ) $\delta 171.20,166.64,164.02,149.31,148.32,141.55,137.24,135.95$, 134.26, 131.04, 130.05, 127.75, 127.02, 126.38, 122.34, 120.56, $61.73,60.53,53.54,35.53,14.38,14.13$; LRMS (ESI): $397[\mathrm{M}+$ $\mathrm{H}]^{+}$; HRMS (ESI) calcd for $\mathrm{C}_{22} \mathrm{H}_{25} \mathrm{~N}_{2} \mathrm{O}_{5}[\mathrm{M}+\mathrm{H}]^{+}$397.1763, found: 397.1756.

(2E,2'E)-Diethyl-3,3'-(2-((S)-3-ethoxy-3-oxo-2-(picolinamido)propyl)1,3-phenylene)diacrylate ( $\left.3 \mathrm{~s}_{d i}\right)$. White solid (56.4 mg, yield $38 \%$ ), mp 138-139 ${ }^{\circ} \mathrm{C} .{ }^{1} \mathrm{H}$ NMR $\left(400 \mathrm{MHz}, \mathrm{CDCl}_{3}\right) \delta 8.55-8.50(\mathrm{~m}, 1 \mathrm{H})$, $8.47(\mathrm{~d}, J=8.7 \mathrm{~Hz}, 1 \mathrm{H}), 8.12(\mathrm{~d}, J=15.7 \mathrm{~Hz}, 2 \mathrm{H}), 8.07-8.00(\mathrm{~m}$, 
1H), 7.82-7.74 (m, 1H), 7.53 (d, $J=7.8 \mathrm{~Hz}, 2 \mathrm{H}), 7.39$ (ddd, $J=$ $7.6,4.8,1.2 \mathrm{~Hz}, 1 \mathrm{H}), 7.31-7.26(\mathrm{~m}, 1 \mathrm{H}), 6.26(\mathrm{~d}, J=15.6 \mathrm{~Hz}, 2 \mathrm{H})$, 4.97-4.83 (m, 1H), 4.28-4.22 (m, 4H), 4.22-4.10 (m, 2H), 3.56 $(\mathrm{dd}, J=14.5,5.6 \mathrm{~Hz}, 1 \mathrm{H}), 3.43(\mathrm{dd}, J=14.4,8.7 \mathrm{~Hz}, 1 \mathrm{H}), 1.34(\mathrm{t}, J$ $=7.1 \mathrm{~Hz}, 6 \mathrm{H}), 1.23(\mathrm{t}, J=7.1 \mathrm{~Hz}, 3 \mathrm{H}) ;{ }^{13} \mathrm{C} \mathrm{NMR}(101 \mathrm{MHz}$, $\left.\mathrm{CDCl}_{3}\right) \delta 171.00,166.44,163.98,149.21,148.28,141.73,137.21$, 135.72, 135.32, 128.78, 127.97, 126.34, 122.30, 122.17, 61.95, 60.72, 53.43, 31.97, 14.45, 14.13; LRMS (ESI): $495[\mathrm{M}+\mathrm{H}]^{+}$; HRMS (ESI) calcd for $\mathrm{C}_{27} \mathrm{H}_{31} \mathrm{~N}_{2} \mathrm{O}_{7}[\mathrm{M}+\mathrm{H}]^{+}$495.2131, found: 495.2128.

(S,E)-Ethyl-3-(2-(3-ethoxy-3-oxo-2-(picolinamido)propyl)-5-methylphenyl)acrylate $\left(3 \boldsymbol{t}_{\text {mono }}\right)$. Pale yellow oil $(44.3 \mathrm{mg}$, yield $36 \%) .{ }^{1} \mathrm{H}$ NMR (400 MHz, $\left.\mathrm{CDCl}_{3}\right) \delta 8.55-8.49(\mathrm{~m}, 1 \mathrm{H}), 8.44(\mathrm{~d}, J=8.4 \mathrm{~Hz}$, $1 \mathrm{H}), 8.14-8.08(\mathrm{~m}, 1 \mathrm{H}), 7.97$ (d, $J=15.8 \mathrm{~Hz}, 1 \mathrm{H}), 7.84-7.75(\mathrm{~m}$, 1H), 7.43-7.33 (m, 2H), 7.14-7.06 (m, 2H), 6.27 (d, $J=15.7 \mathrm{~Hz}$, $1 \mathrm{H}), 5.06-4.93(\mathrm{~m}, 1 \mathrm{H}), 4.22-4.08(\mathrm{~m}, 4 \mathrm{H}), 3.40(\mathrm{dd}, J=14.2$, $6.0 \mathrm{~Hz}, 1 \mathrm{H}), 3.29(\mathrm{dd}, J=14.2,6.7 \mathrm{~Hz}, 1 \mathrm{H}), 2.31(\mathrm{~s}, 3 \mathrm{H}), 1.29(\mathrm{t}, J=$ $7.1 \mathrm{~Hz}, 3 \mathrm{H}), 1.22(\mathrm{t}, J=7.1 \mathrm{~Hz}, 3 \mathrm{H}) ;{ }^{13} \mathrm{C} \mathrm{NMR}\left(101 \mathrm{MHz}, \mathrm{CDCl}_{3}\right)$ $\delta$ 171.24, 166.68, 163.99, 149.36, 148.31, 141.70, 137.28, 137.21, 133.99, 132.96, 130.99, 130.95, 127.57, 126.33, 122.33, 120.20, $61.69,60.46,53.61,35.04,21.15,14.38,14.15$; LRMS (ESI): 411 [M $+\mathrm{H}]^{+}$; HRMS (ESI) calcd for $\mathrm{C}_{23} \mathrm{H}_{27} \mathrm{~N}_{2} \mathrm{O}_{5}[\mathrm{M}+\mathrm{H}]^{+}$411.1920, found: 411.1916 .

(2E,2' E)-Diethyl-3,3'-(2-((S)-3-ethoxy-3-oxo-2-(picolinamido)propyl)5-methyl-1,3-phenylene)diacrylate $\left(\boldsymbol{3 t}_{d i}\right)$. White solid $(68.7 \mathrm{mg}$, yield 45\%), mp 101-102 ${ }^{\circ} \mathrm{C} .{ }^{1} \mathrm{H}$ NMR (400 MHz, $\left.\mathrm{CDCl}_{3}\right) \delta 8.53-$ $8.47(\mathrm{~m}, 1 \mathrm{H}), 8.43(\mathrm{~d}, J=8.6 \mathrm{~Hz}, 1 \mathrm{H}), 8.07$ (d, $J=15.6 \mathrm{~Hz}, 2 \mathrm{H})$, 8.03-7.99 (m, 1H), 7.81-7.71 (m, 1H), 7.40-7.30 (m, 3H), 6.23 (d, $J=15.6 \mathrm{~Hz}, 2 \mathrm{H}), 4.93-4.79(\mathrm{~m}, 1 \mathrm{H}), 4.26-4.08$ (m, 6H), 3.50 (dd, $J=14.5,5.5 \mathrm{~Hz}, 1 \mathrm{H}), 3.37$ (dd, $J=14.5,8.6 \mathrm{~Hz}, 1 \mathrm{H}), 2.30(\mathrm{~s}, 3 \mathrm{H})$, $1.31(\mathrm{t}, J=7.1 \mathrm{~Hz}, 6 \mathrm{H}), 1.23(\mathrm{t}, J=7.1 \mathrm{~Hz}, 3 \mathrm{H}) ;{ }^{13} \mathrm{C} \mathrm{NMR}(101$ $\left.\mathrm{MHz}, \mathrm{CDCl}_{3}\right) \delta 170.95,166.41,163.90,149.16,148.19,141.78$, 137.36, 137.11, 135.42, 132.40, 129.48, 126.23, 122.21, 121.71, 61.82, 60.56, 53.47, 31.46, 21.09, 14.35, 14.04; LRMS (ESI): 509 $[\mathrm{M}+\mathrm{H}]^{+}$; HRMS (ESI) calcd for $\mathrm{C}_{28} \mathrm{H}_{33} \mathrm{~N}_{2} \mathrm{O}_{7}[\mathrm{M}+\mathrm{H}]^{+}$509.2288, found: 509.2281 .

(S,E)-Ethyl-3-(5-chloro-2-(3-ethoxy-3-oxo-2-(picolinamido)propyl) phenyl)acrylate ( $\left.3 \boldsymbol{u}_{\text {mono }}\right)$. Pale yellow oil $(45.4 \mathrm{mg}$, yield $35 \%) .{ }^{1} \mathrm{H}$ NMR (400 MHz, $\mathrm{CDCl}_{3}$ ) $\delta 8.58-8.50(\mathrm{~m}, 1 \mathrm{H}), 8.45(\mathrm{~d}, J=8.4 \mathrm{~Hz}$, $1 \mathrm{H}), 8.10(\mathrm{~d}, J=7.8 \mathrm{~Hz}, 1 \mathrm{H}), 7.91(\mathrm{~d}, J=15.8 \mathrm{~Hz}, 1 \mathrm{H}), 7.84-7.75$ $(\mathrm{m}, 1 \mathrm{H}), 7.50(\mathrm{~d}, J=2.1 \mathrm{~Hz}, 1 \mathrm{H}), 7.40(\mathrm{ddd}, J=7.6,4.8,1.2 \mathrm{~Hz}$, 1H), 7.25-7.20 (m, 1H), 7.19-7.14 (m, 1H), 6.26 (d, $J=15.7 \mathrm{~Hz}$, $1 \mathrm{H}), 5.05-4.95(\mathrm{~m}, 1 \mathrm{H}), 4.25-4.07(\mathrm{~m}, 4 \mathrm{H}), 3.39$ (dd, $J=14.2$, $6.0 \mathrm{~Hz}, 1 \mathrm{H}), 3.28(\mathrm{dd}, J=14.2,6.7 \mathrm{~Hz}, 1 \mathrm{H}), 1.29(\mathrm{t}, J=7.1 \mathrm{~Hz}$, $3 \mathrm{H}), 1.22(\mathrm{t}, J=7.1 \mathrm{~Hz}, 3 \mathrm{H}) ;{ }^{13} \mathrm{C} \mathrm{NMR}\left(101 \mathrm{MHz}, \mathrm{CDCl}_{3}\right) \delta 170.94$, 166.20, 163.99, 149.14, 148.32, 140.20, 137.29, 135.91, 134.36, $133.58,132.36,129.84,126.86,126.45,122.35,121.72,61.87$, 60.67, 53.31, 34.96, 14.32, 14.14; LRMS (ESI): $433\left([\mathrm{M}+\mathrm{H}]^{+}\right.$, $\left.\left(\mathrm{Cl}^{37}\right)\right), 431\left([\mathrm{M}+\mathrm{H}]^{+},\left(\mathrm{Cl}^{35}\right)\right)$; HRMS (ESI) calcd for $\mathrm{C}_{22} \mathrm{H}_{24} \mathrm{ClN}_{2} \mathrm{O}_{5}$ $[\mathrm{M}+\mathrm{H}]^{+}$431.1374, found: 431.1365.

(2E,2' E)-Diethyl-3,3'-(5-chloro-2-((S)-3-ethoxy-3-oxo-2-(picolinamido) propyl)-1,3-phenylene)diacrylate $\left(3 \boldsymbol{u}_{d i}\right)$. White solid $(55.7 \mathrm{mg}$, yield $35 \%)$, mp $83-85{ }^{\circ} \mathrm{C} .{ }^{1} \mathrm{H}$ NMR $\left(400 \mathrm{MHz}, \mathrm{CDCl}_{3}\right) \delta 8.52(\mathrm{~d}, J$ $=4.2 \mathrm{~Hz}, 1 \mathrm{H}), 8.47(\mathrm{~d}, J=8.7 \mathrm{~Hz}, 1 \mathrm{H}), 8.09-7.97(\mathrm{~m}, 3 \mathrm{H}), 7.82-$ $7.76(\mathrm{~m}, 1 \mathrm{H}), 7.53-7.44(\mathrm{~m}, 2 \mathrm{H}), 7.40(\mathrm{ddd}, J=7.5,4.8,1.1 \mathrm{~Hz}$, $1 \mathrm{H}), 6.25(\mathrm{~d}, J=15.6 \mathrm{~Hz}, 2 \mathrm{H}), 4.94-4.82(\mathrm{~m}, 1 \mathrm{H}), 4.30-4.12(\mathrm{~m}$, $6 \mathrm{H}), 3.51(\mathrm{dd}, J=14.5,5.5 \mathrm{~Hz}, 1 \mathrm{H}), 3.38(\mathrm{dd}, J=14.5,8.7 \mathrm{~Hz}$,
1H), $1.34(\mathrm{t}, J=7.1 \mathrm{~Hz}, 6 \mathrm{H}), 1.26(\mathrm{t}, J=7.1 \mathrm{~Hz}, 3 \mathrm{H}) ;{ }^{13} \mathrm{C} \mathrm{NMR}$ $\left(101 \mathrm{MHz}, \mathrm{CDCl}_{3}\right) \delta 170.65,165.93,163.87,148.96,148.18$, $140.34,137.30,137.17,133.70,133.67,128.18,126.31,123.09$, 122.22, 61.96, 60.73, 53.06, 31.60, 14.28, 14.01; LRMS (ESI): 531 $\left([\mathrm{M}+\mathrm{H}]^{+},\left(\mathrm{Cl}^{37}\right)\right), 529\left([\mathrm{M}+\mathrm{H}]^{+},\left(\mathrm{Cl}^{35}\right)\right)$; HRMS (ESI) calcd for $\mathrm{C}_{27} \mathrm{H}_{30} \mathrm{ClN}_{2} \mathrm{O}_{7}[\mathrm{M}+\mathrm{H}]^{+}$529.1742, found: 529.1744 .

(S,E)-Ethyl-2-(picolinamido)-3-(2-styrylphenyl)propanoate (3v). Yellow oil (55.4 mg, 46\%). ${ }^{1} \mathrm{H}$ NMR (400 MHz, $\left.\mathrm{CDCl}_{3}\right) \delta 8.58-$ $8.47(\mathrm{~m}, 1 \mathrm{H}), 8.46-8.37(\mathrm{~m}, 1 \mathrm{H}), 8.00(\mathrm{~d}, J=7.8 \mathrm{~Hz}, 1 \mathrm{H}), 7.72-$ $7.57(\mathrm{~m}, 2 \mathrm{H}), 7.53-7.40(\mathrm{~m}, 3 \mathrm{H}), 7.35-7.12(\mathrm{~m}, 7 \mathrm{H}), 6.90(\mathrm{~d}, J=$ 16.0 Hz, 1H), 5.11-4.93 (m, 1H), 4.24-4.02 (m, 2H), 3.52-3.29 $(\mathrm{m}, 2 \mathrm{H}), 1.16(\mathrm{t}, J=7.1 \mathrm{~Hz}, 3 \mathrm{H}) ;{ }^{13} \mathrm{C} \mathrm{NMR}\left(101 \mathrm{MHz}, \mathrm{CDCl}_{3}\right)$ $\delta 171.55,164.09,149.28,148.16,137.47,137.16,137.11,134.30$, 130.96, 130.80, 128.68, 127.70, 127.68, 127.63, 126.77, 126.27, 126.06, 125.70, 122.29, 61.65, 53.79, 35.89, 14.13; LRMS (ESI): $401[\mathrm{M}+\mathrm{H}]^{+}$; HRMS (ESI) calcd for $\mathrm{C}_{25} \mathrm{H}_{25} \mathrm{~N}_{2} \mathrm{O}_{3}[\mathrm{M}+\mathrm{H}]^{+}$ 401.1865, found: 401.1860 .

(E)-Methyl-3-(3-methyl-2-(2-(picolinamido)ethyl)phenyl)acrylate (5a). Pale yellow oil (86.6 mg, yield 89\%). ${ }^{1} \mathrm{H}$ NMR $(400 \mathrm{MHz}$, CDCl3) $\delta$ 8.59-8.49 (m, 1H), 8.31-8.17 (m, 2H), $8.11(\mathrm{~d}, J=$ $15.7 \mathrm{~Hz}, 1 \mathrm{H}), 7.90-7.82(\mathrm{~m}, 1 \mathrm{H}), 7.46-7.38(\mathrm{~m}, 2 \mathrm{H}), 7.23(\mathrm{~d}, J=$ $7.1 \mathrm{~Hz}, 1 \mathrm{H}), 7.20-7.13(\mathrm{~m}, 1 \mathrm{H}), 6.31$ (d, $J=15.7 \mathrm{~Hz}, 1 \mathrm{H}), 3.77(\mathrm{~s}$, 3H), 3.63-3.55 (m, 2H), 3.18-3.09 (m, 2H), 2.46 (s, 3H); ${ }^{13} \mathrm{C}$ NMR (101 MHz, CDCl3) $\delta 167.17,164.44,149.80,148.02,142.76$, 137.64, 137.24, 136.68, 134.04, 132.29, 126.83, 126.10, 124.79, 122.10, 120.06, 51.61, 39.40, 29.35, 19.94; LRMS (ESI): $325[\mathrm{M}+$ $\mathrm{H}]^{+}$; HRMS (ESI) calcd for $\mathrm{C}_{19} \mathrm{H}_{21} \mathrm{~N}_{2} \mathrm{O}_{3}[\mathrm{M}+\mathrm{H}]^{+}$325.1552, found: 325.1548 .

(E)-Ethyl-3-(3-methyl-2-(2-(picolinamido)ethyl)phenyl)acrylate (5b). Pale yellow oil (87.2 mg, yield 86\%). ${ }^{1} \mathrm{H}$ NMR $(400 \mathrm{MHz}$, CDCl3) $\delta$ 8.59-8.49 (m, 1H), 8.26-8.16 (m, 2H), $8.10(\mathrm{~d}, J=$ $15.7 \mathrm{~Hz}, 1 \mathrm{H}), 7.89-7.80(\mathrm{~m}, 1 \mathrm{H}), 7.46-7.38(\mathrm{~m}, 2 \mathrm{H}), 7.23(\mathrm{~d}, J=$ $7.1 \mathrm{~Hz}, 1 \mathrm{H}), 7.19-7.14(\mathrm{~m}, 1 \mathrm{H}), 6.31$ (d, $J=15.7 \mathrm{~Hz}, 1 \mathrm{H}), 4.23$ (q, $J=7.1 \mathrm{~Hz}, 2 \mathrm{H}), 3.63-3.54(\mathrm{~m}, 2 \mathrm{H}), 3.18-3.09(\mathrm{~m}, 2 \mathrm{H}), 2.46$ $(\mathrm{s}, 3 \mathrm{H}), 1.33(\mathrm{t}, J=7.1 \mathrm{~Hz}, 3 \mathrm{H}) ;{ }^{13} \mathrm{C} \mathrm{NMR}(101 \mathrm{MHz}, \mathrm{CDCl} 3)$ $\delta$ 166.92, 164.53, 149.92, 148.13, 142.62, 137.75, 137.37, $136.73,134.25,132.32,126.93,126.19,124.92,122.22,120.68$, 60.55, 39.50, 29.49, 20.06, 14.39; LRMS (ESI): $339[\mathrm{M}+\mathrm{H}]^{+}$; HRMS (ESI) calcd for $\mathrm{C}_{20} \mathrm{H}_{23} \mathrm{~N}_{2} \mathrm{O}_{3}[\mathrm{M}+\mathrm{H}]^{+} 339.1709$, found: 339.1706 .

(E)-N-(2-Methyl-6-(3-oxopent-1-en-1-yl)phenethyl)picolinamide (5c). White solid (89.5 mg, yield 93\%), mp 86-87 ${ }^{\circ} \mathrm{C} .{ }^{1} \mathrm{H}$ NMR (400 MHz, CDCl3) $\delta 8.57-8.47(\mathrm{~m}, 1 \mathrm{H}), 8.31-8.22(\mathrm{~m}, 1 \mathrm{H}), 8.20$ $(\mathrm{d}, J=7.8 \mathrm{~Hz}, 1 \mathrm{H}), 8.04(\mathrm{~d}, J=16.0 \mathrm{~Hz}, 1 \mathrm{H}), 7.90-7.82(\mathrm{~m}, 1 \mathrm{H})$, 7.47-7.40 (m, 2H), $7.23(\mathrm{~d}, J=7.1 \mathrm{~Hz}, 1 \mathrm{H}), 7.20-7.14(\mathrm{~m}, 1 \mathrm{H})$, $6.57(\mathrm{~d}, J=16.0 \mathrm{~Hz}, 1 \mathrm{H}), 3.63-3.55(\mathrm{~m}, 2 \mathrm{H}), 3.19-3.11(\mathrm{~m}, 2 \mathrm{H})$, $2.72(\mathrm{q}, J=7.3 \mathrm{~Hz}, 2 \mathrm{H}), 2.45(\mathrm{~s}, 3 \mathrm{H}), 1.13(\mathrm{t}, J=7.3 \mathrm{~Hz}, 3 \mathrm{H}) ;{ }^{13} \mathrm{C}$ NMR (101 MHz, CDCl3) $\delta$ 201.28, 164.66, 149.88, 148.19, $140.41,137.68,137.50,137.01,134.60,132.43$, 128.82, 127.02, 126.34, 124.92, 122.27, 39.56, 33.64, 29.51, 20.11, 8.32; LRMS (ESI): $323[\mathrm{M}+\mathrm{H}]^{+}$; HRMS (ESI) calcd for $\mathrm{C}_{20} \mathrm{H}_{23} \mathrm{~N}_{2} \mathrm{O}_{2}[\mathrm{M}+\mathrm{H}]^{+}$ 323.1760, found: 323.1762.

(E)-Methyl-3-(3-methyl-2-(2-(picolinamido)propyl)phenyl)acrylate (5d). Pale yellow oil (91.4 mg, yield 90\%). ${ }^{1} \mathrm{H}$ NMR (400 MHz, CDCl3) $\delta 8.57-8.48(\mathrm{~m}, 1 \mathrm{H}), 8.19(\mathrm{~d}, J=15.7 \mathrm{~Hz}, 1 \mathrm{H}), 8.14(\mathrm{~d}, J=$ $7.8 \mathrm{~Hz}, 1 \mathrm{H}), 8.04$ (d, $J=8.3 \mathrm{~Hz}, 1 \mathrm{H}), 7.87-7.78$ (m, 1H), 7.41 (ddd, $J=7.6,4.8,1.2 \mathrm{~Hz}, 1 \mathrm{H}), 7.37(\mathrm{~d}, J=7.6 \mathrm{~Hz}, 1 \mathrm{H}), 7.23-7.19(\mathrm{~m}$, 
1H), 7.17-7.10 (m, 1H), $6.25(\mathrm{~d}, J=15.7 \mathrm{~Hz}, 1 \mathrm{H}), 4.45-4.31(\mathrm{~m}$, $1 \mathrm{H}), 3.81$ (s, 3H), 3.21 (dd, $J=13.9,6.6 \mathrm{~Hz}, 1 \mathrm{H}), 2.99$ (dd, $J=13.9$, $8.0 \mathrm{~Hz}, 1 \mathrm{H}), 2.49$ (s, 3H), 1.25 (d, $J=6.7 \mathrm{~Hz}, 3 \mathrm{H}) ;{ }^{13} \mathrm{C}$ NMR (101 $\mathrm{MHz}, \mathrm{CDCl} 3) \delta 167.47,163.58,149.98,148.01,143.81,138.04$, $137.46,136.70,134.64,132.44,126.89,126.16,124.95,122.28$, 119.93, 51.79, 46.35, 36.08, 20.42, 20.36; LRMS (ESI): $339[\mathrm{M}+$ $\mathrm{H}]^{+}$; HRMS (ESI) calcd for $\mathrm{C}_{20} \mathrm{H}_{23} \mathrm{~N}_{2} \mathrm{O}_{3}[\mathrm{M}+\mathrm{H}]^{+} 339.1709$, found: 339.1708.

(E)-Ethyl-3-(3-methyl-2-(2-(picolinamido)propyl)phenyl)acrylate (5e). Pale yellow oil (93.4 mg, yield 88\%). ${ }^{1} \mathrm{H}$ NMR $(400 \mathrm{MHz}$, CDCl3) $\delta 8.57-8.49(\mathrm{~m}, 1 \mathrm{H}), 8.18(\mathrm{~d}, J=15.7 \mathrm{~Hz}, 1 \mathrm{H}), 8.14(\mathrm{~d}, J=$ $7.8 \mathrm{~Hz}, 1 \mathrm{H}), 8.05$ (d, $J=8.3 \mathrm{~Hz}, 1 \mathrm{H}), 7.86-7.78(\mathrm{~m}, 1 \mathrm{H}), 7.43-$ $7.34(\mathrm{~m}, 2 \mathrm{H}), 7.23-7.17(\mathrm{~m}, 1 \mathrm{H}), 7.16-7.10(\mathrm{~m}, 1 \mathrm{H}), 6.25(\mathrm{~d}, J=$ $15.7 \mathrm{~Hz}, 1 \mathrm{H}), 4.44-4.32(\mathrm{~m}, 1 \mathrm{H}), 4.31-4.23(\mathrm{~m}, 2 \mathrm{H}), 3.21$ (dd, $J=$ 13.9, 6.6 Hz, 1H), 2.99 (dd, $J=13.9,8.0 \mathrm{~Hz}, 1 \mathrm{H}), 2.49$ (s, 3H), $1.35(\mathrm{t}, J=7.1 \mathrm{~Hz}, 3 \mathrm{H}), 1.25(\mathrm{~d}, J=6.7 \mathrm{~Hz}, 3 \mathrm{H}) ;{ }^{13} \mathrm{C} \mathrm{NMR}(101$ $\mathrm{MHz}, \mathrm{CDCl} 3) \delta 167.08,163.55,149.96,147.99,143.54,138.00$, 137.48, 136.66, 134.71, 132.37, 126.87, 126.16, 124.95, 122.29, 120.38, 60.57, 46.35, 36.08, 20.42, 20.37, 14.47; LRMS (ESI): 353 $[\mathrm{M}+\mathrm{H}]^{+}$; HRMS (ESI) calcd for $\mathrm{C}_{21} \mathrm{H}_{25} \mathrm{~N}_{2} \mathrm{O}_{3}[\mathrm{M}+\mathrm{H}]^{+}$353.1865, found: 353.1860 .

(E)-N-(1-(2-Methyl-6-(3-oxopent-1-en-1-yl)phenyl)propan-2-yl) picolinamide (5f). White solid (91.6 mg, yield 91\%), $\mathrm{mp} 75-76{ }^{\circ} \mathrm{C}$. ${ }^{1} \mathrm{H}$ NMR (400 MHz, CDCl3) $\delta$ 8.57-8.48 (m, 1H), 8.20-8.10 (m, $2 \mathrm{H}), 8.04(\mathrm{~d}, J=8.0 \mathrm{~Hz}, 1 \mathrm{H}), 7.88-7.79(\mathrm{~m}, 1 \mathrm{H}), 7.45-7.39(\mathrm{~m}$, 2H), 7.24-7.18 (m, 1H), 7.18-7.11 (m, 1H), $6.56(\mathrm{~d}, J=15.9 \mathrm{~Hz}$, $1 \mathrm{H}), 4.41-4.29(\mathrm{~m}, 1 \mathrm{H}), 3.28$ (dd, $J=13.9,6.2 \mathrm{~Hz}, 1 \mathrm{H}), 2.96(\mathrm{dd}, J$ $=13.9,8.4 \mathrm{~Hz}, 1 \mathrm{H}), 2.90-2.71(\mathrm{~m}, 2 \mathrm{H}), 2.48(\mathrm{~s}, 3 \mathrm{H}), 1.25(\mathrm{~d}, J=$ $6.7 \mathrm{~Hz}, 3 \mathrm{H}), 1.18$ (t, $J=7.3 \mathrm{~Hz}, 3 \mathrm{H}) ;{ }^{13} \mathrm{C} \mathrm{NMR}(101 \mathrm{MHz}, \mathrm{CDCl} 3)$ $\delta 201.49,163.63,149.96,148.07,141.14,137.98,137.51,136.93$, $134.81,132.45,128.59,126.91,126.25,124.93,122.28,46.65$, 35.97, 33.64, 20.64, 20.07, 8.39; LRMS (ESI): 337 [M + H $]^{+}$; HRMS (ESI) calcd for $\mathrm{C}_{21} \mathrm{H}_{25} \mathrm{~N}_{2} \mathrm{O}_{2}[\mathrm{M}+\mathrm{H}]^{+} 337.1916$, found: 337.1914.

(E)-Methyl-3-(3-fluoro-2-(2-(picolinamido)ethyl)phenyl)acrylate (5g). White solid (80.8 mg, yield $82 \%$ ), mp 77-79 ${ }^{\circ} \mathrm{C} .{ }^{1} \mathrm{H}$ NMR (400 MHz, CDCl3) $\delta 8.53-8.46(\mathrm{~m}, 1 \mathrm{H}), 8.23-8.08(\mathrm{~m}, 2 \mathrm{H}), 7.98$ $(\mathrm{d}, J=15.8 \mathrm{~Hz}, 1 \mathrm{H}), 7.87-7.79(\mathrm{~m}, 1 \mathrm{H}), 7.40$ (ddd, $J=7.6,4.8$, $1.1 \mathrm{~Hz}, 1 \mathrm{H}), 7.35$ (d, $J=7.8 \mathrm{~Hz}, 1 \mathrm{H}), 7.26-7.20(\mathrm{~m}, 1 \mathrm{H}), 7.13-$ $7.02(\mathrm{~m}, 1 \mathrm{H}), 6.30$ (d, $J=15.8 \mathrm{~Hz}, 1 \mathrm{H}), 3.74(\mathrm{~s}, 3 \mathrm{H}), 3.70-3.64$ $(\mathrm{m}, 2 \mathrm{H}), 3.17-3.12(\mathrm{~m}, 2 \mathrm{H}) ;{ }^{13} \mathrm{C}$ NMR (101 $\left.\mathrm{MHz}, \mathrm{CDCl}_{3}\right)$ $\delta 166.83,164.45,161.72\left(\mathrm{~d}, J_{\mathrm{C}-\mathrm{F}}=245.2 \mathrm{~Hz}\right), 149.86,148.05$, $140.90\left(\mathrm{~d}, J_{\mathrm{C}-\mathrm{F}}=3.5 \mathrm{~Hz}\right), 137.41,136.24\left(\mathrm{~d}, J_{\mathrm{C}-\mathrm{F}}=4.5 \mathrm{~Hz}\right)$, $128.28\left(\mathrm{~d}, J_{\mathrm{C}-\mathrm{F}}=9.1 \mathrm{~Hz}\right), 126.21,126.02\left(\mathrm{~d}, J_{\mathrm{C}-\mathrm{F}}=16.1 \mathrm{~Hz}\right)$, $122.60\left(\mathrm{~d}, J_{\mathrm{C}-\mathrm{F}}=3.3 \mathrm{~Hz}\right), 122.34,121.33,116.68\left(\mathrm{~d}, J_{\mathrm{C}-\mathrm{F}}=23.3\right.$ $\mathrm{Hz}$ ), 51.85, 39.72, 25.44; LRMS (ESI): 329 [M + H] $]^{+}$; HRMS (ESI) calcd for $\mathrm{C}_{18} \mathrm{H}_{18} \mathrm{FN}_{2} \mathrm{O}_{3}[\mathrm{M}+\mathrm{H}]^{+} 329.1301$, found: 329.1296 .

(E)-Methyl-3-(3-chloro-2-(2-(picolinamido)ethyl)phenyl)acrylate (5h). White solid (81.6 mg, yield 79\%), mp 93-95 ${ }^{\circ} \mathrm{C} .{ }^{1} \mathrm{H}$ NMR (400 MHz, CDCl3) $\delta$ 8.53-8.47 (m, 1H), 8.21-8.09 (m, 2H), 8.04 $(\mathrm{d}, J=15.7 \mathrm{~Hz}, 1 \mathrm{H}), 7.87-7.80(\mathrm{~m}, 1 \mathrm{H}), 7.47-7.37(\mathrm{~m}, 3 \mathrm{H}), 7.24-$ $7.16(\mathrm{~m}, 1 \mathrm{H}), 6.26(\mathrm{~d}, J=15.7 \mathrm{~Hz}, 1 \mathrm{H}), 3.74(\mathrm{~s}, 3 \mathrm{H}), 3.72-3.67$ $(\mathrm{m}, 2 \mathrm{H}), 3.29(\mathrm{t}, J=7.2 \mathrm{~Hz}, 2 \mathrm{H}) ;{ }^{13} \mathrm{C}$ NMR (101 MHz, CDCl3) $\delta 166.74,164.52,149.89,148.09,141.81,137.36,136.42,136.16$, 135.76, 131.10, 128.10, 126.21, 125.70, 122.31, 121.67, 51.86, 39.00, 29.99; LRMS (ESI): $347\left([\mathrm{M}+\mathrm{H}]^{+},\left(\mathrm{Cl}^{37}\right)\right), 345\left([\mathrm{M}+\mathrm{H}]^{+}\right.$, $\left(\mathrm{Cl}^{35}\right)$ ); HRMS (ESI) calcd for $\mathrm{C}_{18} \mathrm{H}_{18} \mathrm{ClN}_{2} \mathrm{O}_{3}[\mathrm{M}+\mathrm{H}]^{+}$345.1006, found: 345.1003 .
(E)-Methyl-3-(2-(2-(picolinamido)ethyl)phenyl)acrylate (5i $\left.{ }_{\text {mono }}\right)$. Pale yellow oil (50.3 mg, yield 54\%). ${ }^{1} \mathrm{H}$ NMR (400 MHz, CDCl3) $\delta$ 8.54-8.45 (m, 1H), 8.23-8.09 (m, 2H), 8.04 (d, $J=15.8 \mathrm{~Hz}, 1 \mathrm{H})$, $7.88-7.79(\mathrm{~m}, 1 \mathrm{H}), 7.58$ (d, $J=7.9 \mathrm{~Hz}, 1 \mathrm{H}), 7.41$ (ddd, $J=7.6,4.8$, $1.2 \mathrm{~Hz}, 1 \mathrm{H}), 7.37-7.31(\mathrm{~m}, 1 \mathrm{H}), 7.30-7.23(\mathrm{~m}, 2 \mathrm{H}), 6.35(\mathrm{~d}, J=$ $15.8 \mathrm{~Hz}, 1 \mathrm{H}), 3.77(\mathrm{~s}, 3 \mathrm{H}), 3.72-3.65(\mathrm{~m}, 2 \mathrm{H}), 3.10(\mathrm{t}, J=7.3 \mathrm{~Hz}$, $2 \mathrm{H}) ;{ }^{13} \mathrm{C}$ NMR (101 MHz, CDCl3) $\delta 167.28,164.42,149.88$, $148.10,141.93$, 138.66, 137.45, 133.61, 130.65, 130.41, 127.33, 126.95, 126.25, 122.32, 119.94, 51.82, 40.69, 33.27; LRMS (ESI): $311[\mathrm{M}+\mathrm{H}]^{+}$; HRMS (ESI) calcd for $\mathrm{C}_{18} \mathrm{H}_{19} \mathrm{~N}_{2} \mathrm{O}_{3}[\mathrm{M}+\mathrm{H}]^{+}$ 311.1396, found: 311.1397.

(2E,2'E)-Dimethyl-3,3'-(2-(2-(picolinamido)ethyl)-1,3-phenylene) diacrylate $\left(\boldsymbol{5 i}_{d i}\right)$. White solid $(35.5 \mathrm{mg}$, yield $30 \%)$, mp 106$107{ }^{\circ} \mathrm{C} .{ }^{1} \mathrm{H}$ NMR $\left(400 \mathrm{MHz}, \mathrm{CDCl}_{3}\right) \delta 8.50-8.44(\mathrm{~m}, 1 \mathrm{H}), 8.16(\mathrm{~d}, J$ $=7.8 \mathrm{~Hz}, 1 \mathrm{H}), 8.14-8.06(\mathrm{~m}, 3 \mathrm{H}), 7.83-7.78(\mathrm{~m}, 1 \mathrm{H}), 7.56(\mathrm{~d}, J=$ $7.8 \mathrm{~Hz}, 2 \mathrm{H}$ ), 7.39 (ddd, $J=7.6,4.8,1.2 \mathrm{~Hz}, 1 \mathrm{H}), 7.31-7.27(\mathrm{~m}$, $1 \mathrm{H}), 6.29$ (d, $J=15.7 \mathrm{~Hz}, 2 \mathrm{H}), 3.76(\mathrm{~s}, 6 \mathrm{H}), 3.63-3.57(\mathrm{~m}, 2 \mathrm{H})$, 3.28-3.20 (m, 2H); $\left.{ }^{13} \mathrm{C} \mathrm{NMR} \mathrm{(101} \mathrm{MHz,} \mathrm{CDCl}_{3}\right) \delta 166.92,164.52$, 149.77, 148.06, 142.07, 137.60, 137.28, 135.22, 128.76, 127.54, 126.19, 122.28, 121.42, 51.85, 40.23, 28.93; LRMS (ESI): $395[\mathrm{M}+$ $\mathrm{H}]^{+}$; HRMS (ESI) calcd for $\mathrm{C}_{22} \mathrm{H}_{23} \mathrm{~N}_{2} \mathrm{O}_{5}[\mathrm{M}+\mathrm{H}]^{+}$395.1607, found: 395.1601 .

Compounds $3 v-1,5 c-1,5 c-2$ and $5 c-3$ were prepared according to similar procedures described in literature: Y.-S. Zhao and G. Chen, Org. Lett., 2011, 13, 4850-4853

(S,E)-2-((tert-Butoxycarbonyl)amino)-3-(2-styrylphenyl)propanoic acid (3v-1). Yellow oil (41.8 mg, 82\%). ${ }^{1} \mathrm{H}$ NMR (400 MHz, DMSO) $\delta 12.61(\mathrm{~s}, 1 \mathrm{H}), 7.71(\mathrm{~d}, J=7.7 \mathrm{~Hz}, 1 \mathrm{H}), 7.68-7.60(\mathrm{~m}, 2 \mathrm{H}), 7.56(\mathrm{~d}$, $J=16.2 \mathrm{~Hz}, 1 \mathrm{H}), 7.42-7.36(\mathrm{~m}, 2 \mathrm{H}), 7.32-7.20(\mathrm{~m}, 5 \mathrm{H}), 7.17(\mathrm{~d}, J$ $=16.3 \mathrm{~Hz}, 1 \mathrm{H}), 4.14-4.04(\mathrm{~m}, 1 \mathrm{H}), 3.29(\mathrm{dd}, J=14.1,4.2 \mathrm{~Hz}, 1 \mathrm{H})$, $2.96(\mathrm{dd}, J=14.0,10.0 \mathrm{~Hz}, 1 \mathrm{H}), 1.31(\mathrm{~s}, 9 \mathrm{H}) ;{ }^{13} \mathrm{C} \mathrm{NMR}(101 \mathrm{MHz}$, DMSO) $\delta 173.62,155.53,137.31,136.01,135.73,130.77,130.12$, 128.67, 127.72, 127.34, 126.96, 126.64, 125.38, 125.29, 78.10, 54.92, 34.23, 28.13; LRMS (ESI): 390 [M + Na $]^{+}$; HRMS (ESI) calcd for $\mathrm{C}_{22} \mathrm{H}_{25} \mathrm{NO}_{4} \mathrm{Na}[\mathrm{M}+\mathrm{Na}]^{+}$390.1681, found: 390.1675 .

(E)-tert-Butyl-2-methyl-6-(3-oxopent-1-en-1-yl)phenethyl(picolinoyl)carbamate (5c-1). Pale yellow oil (115.4 mg, yield 91\%). ${ }^{1} \mathrm{H}$ NMR (400 MHz, CDCl3) $\delta 8.62-8.55(\mathrm{~m}, 1 \mathrm{H}), 8.23(\mathrm{~d}, J=16.2 \mathrm{~Hz}, 1 \mathrm{H})$, $7.86-7.79(\mathrm{~m}, 1 \mathrm{H}), 7.68(\mathrm{~d}, J=7.8 \mathrm{~Hz}, 1 \mathrm{H}), 7.46(\mathrm{~d}, J=7.3 \mathrm{~Hz}, 1 \mathrm{H})$, 7.41 (ddd, $J=7.6,4.8,1.2 \mathrm{~Hz}, 1 \mathrm{H}), 7.24-7.13$ (m, 2H), 6.60 (d, $J=$ $16.2 \mathrm{~Hz}, 1 \mathrm{H}), 3.99-3.88(\mathrm{~m}, 2 \mathrm{H}), 3.26-3.15(\mathrm{~m}, 2 \mathrm{H}), 2.96$ (q, $J=$ $7.3 \mathrm{~Hz}, 2 \mathrm{H}), 2.49(\mathrm{~s}, 3 \mathrm{H}), 1.19(\mathrm{t}, J=7.3 \mathrm{~Hz}, 3 \mathrm{H}), 1.15(\mathrm{~s}, 9 \mathrm{H}) ;{ }^{13} \mathrm{C}$ NMR (101 MHz, CDCl3) $\delta$ 202.57, 171.55, 154.83, 153.06, 148.23, 140.97 , 138.02, 137.13, 136.16, 134.78, 132.28, 129.85, 127.12, 125.39, 124.68, 122.89, 83.39, 45.14, 32.22, 28.57, 27.43, 19.96, 8.64; LRMS (ESI): $445[\mathrm{M}+\mathrm{Na}]^{+}$; HRMS (ESI) calcd for $\mathrm{C}_{25} \mathrm{H}_{30} \mathrm{~N}_{2} \mathrm{O}_{4} \mathrm{Na}[\mathrm{M}$ $+\mathrm{Na}]^{+}$445.2103, found: 445.2099.

tert-Butyl-5-methyl-1-(2-oxobutyl)-3,4-dihydroisoquinoline-2(1H)carboxylate (5c-2). White solid (76.3 mg, yield 88\%), mp 104$106{ }^{\circ} \mathrm{C} .{ }^{1} \mathrm{H}$ NMR $\left(400 \mathrm{MHz}, \mathrm{CDCl}_{3}\right) \delta 7.20-6.92(\mathrm{~m}, 3 \mathrm{H}), 5.72-5.48$ (m, 1H), 4.36-3.97 (m, 1H), 3.38-3.12 (m, 1H), 2.98-2.38 (m, 6H), $2.23(\mathrm{~s}, 3 \mathrm{H}), 1.45(\mathrm{~s}, 9 \mathrm{H}), 1.06$ (t, $J=7.2 \mathrm{~Hz}, 3 \mathrm{H}) ;{ }^{13} \mathrm{C}$ NMR (126 $\mathrm{MHz}, \mathrm{CDCl}_{3}$, rotamer peaks exist in the spectra) $\delta 209.02,208.59$, $154.58,154.36,137.13$, 136.96, 136.82, 136.61, 132.94, 132.78, 128.33, 126.04, 124.87, 124.64, 80.42, 80.01, 51.90, 51.39, 50.00, 49.84, 38.06, 37.15, 36.96, 35.87, 28.47, 25.98, 25.77, 19.48, 7.76; 
LRMS (ESI): 340 [M + Na] $]^{+}$; HRMS (ESI) calcd for $\mathrm{C}_{19} \mathrm{H}_{27} \mathrm{NO}_{3} \mathrm{Na}$ $[\mathrm{M}+\mathrm{Na}]^{+} 340.1889$, found: 340.1884 .

tert-Butyl-2-methyl-6-(3-propionyloxiran-2-yl)phenethylcarbamate (5c-3). White solid (8.3 mg, yield 9\%), mp 90-91 ${ }^{\circ} \mathrm{C} .{ }^{1} \mathrm{H}$ NMR (400 $\left.\mathrm{MHz}, \mathrm{CDCl}_{3}\right) \delta$ 7.21-7.00 (m, 3H), 4.85-4.71 (m, 1H), 4.48-4.36 (m, 1H), 3.45-3.29 (m, 1H), 3.27-3.15 (m, 2H), 2.98-2.83 (m, 2H), $2.62(\mathrm{q}, J=7.2 \mathrm{~Hz}, 2 \mathrm{H}), 2.37(\mathrm{~s}, 3 \mathrm{H}), 1.42(\mathrm{~s}, 9 \mathrm{H}), 1.12(\mathrm{t}, J=$ $7.2 \mathrm{~Hz}, 3 \mathrm{H}) ;{ }^{13} \mathrm{C} \mathrm{NMR}\left(101 \mathrm{MHz}, \mathrm{CDCl}_{3}\right) \delta 207.49,156.05,137.00$, 135.54, 134.69, 130.73, 126.97, 122.53, 79.50, 62.99, 56.29, 40.26, 30.63, 30.15, 28.50, 19.56, 7.04; LRMS (ESI): 356 [M + Na $]^{+}$; HRMS (ESI) calcd for $\mathrm{C}_{19} \mathrm{H}_{27} \mathrm{NO}_{4} \mathrm{Na}[\mathrm{M}+\mathrm{Na}]^{+} 356.1838$, found: 356.1832 .

\section{Acknowledgements}

We gratefully acknowledge financial support from the National Natural Science Foundation of China (No. 21602022), the Open Project Program of Antibiotics Research and Re-evaluation Key Laboratory of Sichuan Province (No. ARRLKF15-01), Chengdu University New Faculty Start-up Funding (No. 2081915037) and Zhejiang Provincial Natural Science Foundation (No. LQ14B020004).

\section{Notes and references}

1 For recent reviews: (a) J. Wencel-Delord and F. Glorius, Nat. Chem., 2013, 5, 369-375; (b) M.-L. Louillat and F. W. Patureau, Chem. Soc. Rev., 2014, 43, 901-910; (c) L. Ackermann, R. Vicente and A. R. Kapdi, Angew. Chem., Int. Ed., 2009, 48, 9792-9826; (d) J. Wencel-Delord, T. Droge, F. Liu and F. Glorius, Chem. Soc. Rev., 2011, 40, 4740-4761; (e) O. Daugulis, H.-Q. Do and D. Shabashov, Acc. Chem. Res., 2009, 42, 1074-1086; $(f)$ D. A. Colby, R. G. Bergman and J. A. Ellman, Chem. Rev., 2010, 110, 624-655; $(g)$ G. Rouquet and N. Chatani, Angew. Chem., Int. Ed., 2013, 52, 1172611743; (h) K. Godula and D. Sames, Science, 2006, 312, 6772; (i) S. H. Cho, J. Y. Kim, J. Kwak and S. Chang, Chem. Soc. Rev., 2011, 40, 5068-5083; (j) B.-J. Li and Z.-J. Shi, Chem. Soc. Rev., 2012, 41, 5588-5598; (k) S. Messaoudi, J.-D. Brion and M. Alami, Eur. J. Org. Chem., 2010, 64956516; (l) K. M. Engle, T.-S. Mei, M. Wasa and J.-Q. Yu, Acc. Chem. Res., 2012, 45, 788-802; $(\mathrm{m})$ L. F. McMurray and M. J. Gaunt, Chem. Soc. Rev., 2011, 40, 1885-1898; (n) F. Kakiuchi and S. Murai, Acc. Chem. Res., 2002, 35, 826834; (o) X. Chen, K. M. Engle, D.-H. Wang and J.-Q. Yu, Angew. Chem., Int. Ed., 2009, 48, 5094-5115; (p) C.-L. Sun, B.-J. Li and Z.-J. Shi, Chem. Commun., 2010, 677-685; (q) J. Yamaguchi, A. D. Yamaguchi and K. Itami, Angew. Chem., Int. Ed., 2012, 51, 8960-9009; $(r)$ B. Li and P. H. Dixneuf, Chem. Soc. Rev., 2013, 42, 5744-5767; (s) D. Alberico, M. E. Scott and M. Lautens, Chem. Rev., 2007, 107, 174-238; $(t)$ T. W. Lyons and M. S. Sanford, Chem. Rev., 2010, 110, 1147-1169; (u) I. V. Seregin and V. Gevorgyan, Chem. Soc. Rev., 2007, 36, 1173-1193; (v) Y.-Y. Ping, Q.-P. Ding and Y.-Y. Peng, ACS Catal., 2016, 6, 5989-6005; (w) M. Gulías and J. L. Mascareñas, Angew. Chem., Int. Ed., 2016, 55, 11000-11019; (x) M. P. Drapeau and L. J. Gooßen, Chem.Eur. J., 2016, 22, 18654-18677; (y) D.-H. Wei, X.-J. Zhu,
J.-L. Niu and M.-P. Song, ChemCatChem, 2016, 8, 12421263; ( $z$ ) Z. Chen, B. Wang, J. Zhang, W. Yu, Z. Liu and Y. Zhang, Org. Chem. Front., 2015, 2, 1107-1295.

2 For selected papers: (a) E. T. Nadres and O. Daugulis, J. Am. Chem. Soc., 2012, 134, 7-10; (b) G. He, S.-Y. Zhang, W. A. Nack, Q. Li and G. Chen, Angew. Chem., Int. Ed., 2013, 52, 11124-11128; (c) G. Shan, X.-L. Yang, Y. Zong and Y. Rao, Angew. Chem., Int. Ed., 2013, 52, 13606-13610; (d) Q. Wang, J. Han, C. Wang, J.-Y. Zhang, Z.-B. Huang, D.-Q. Shi and Y.-S. Zhao, Chem. Sci., 2014, 5, 4962-4967; (e) A. Deb, S. Bag, R. Kancherla and D. Maiti, J. Am. Chem. Soc., 2014, 136, 13602-13605; $(f)$ H. Shiota, Y. Ano, Y. Aihara, Y. Fukumoto and N. Chatani, J. Am. Chem. Soc., 2011, 133, 14952-14955; (g) Y.-J. Liu, H. Xu, W.-J. Kong, M. Shang, H.-X. Dai and J.-Q. Yu, Nature, 2014, 515, 389-393; (h) W.-B. Yang, S.-Q. Ye, D. Fanning, T. Coon, Y. Schmidt, P. Krenitsky, D. Stamos and J.-Q. Yu, Angew. Chem., Int. Ed., 2015, 54, 2501-2504; (i) S.-Y. Yan, Y.-J. Liu, B. Liu, Y.-H. Liu and B.-F. Shi, Chem. Commun., 2015, 51, 4069-4072; (j) Q. Zhang, X.-S. Yin, K. Chen, S.-Q. Zhang and B.-F. Shi, J. Am. Chem. Soc., 2015, 137, 8219-8226; (k) D. Shabashov and O. Daugulis, J. Am. Chem. Soc., 2010, 132, 3965-3972; (l) V. G. Zaitsev, D. Shabashov and O. Daugulis, J. Am. Chem. Soc., 2005, 127, 13154-13155; (m) G. Li, L. Wan, G.-F. Zhang, D. Leow, J. Spangler and J.-Q. Yu, J. Am. Chem. Soc., 2015, 137, 4391-4397; (n) L.-S. Zhang, G.-H. Chen, X. Wang, Q.-Y. Guo, X.-S. Zhang, F. Pan, K. Chen and Z.-J. Shi, Angew. Chem., Int. Ed., 2014, 53, 3899-3903; (o) P. Becker, D. L. Priebbenow, R. Pirwerdjan and C. Bolm, Angew. Chem., Int. Ed., 2014, 53, 269-271; (p) F. W. Patureau, T. Besset and F. Glorius, Angew. Chem., Int. $E d$., 2011, 50, 1064-1067; (q) K. M. Engle, D.-H. Wang and J.-Q. Yu, Angew. Chem., Int. Ed., 2010, 49, 6169-6173; (r) M. Castaing, S. L. Wason, B. Estepa, J. F. Hooper and M. C. Willis, Angew. Chem., Int. Ed., 2013, 52, 13280-13283; $(s)$ L. Grigorjeva and O. Daugulis, Angew. Chem., Int. Ed., 2014, 53, 10209-10212; $(t)$ L. Yang, G.-Y. Zhang and H.-M. Huang, Adv. Synth. Catal., 2014, 356, 1509-1515; (u) K. Parthasarathy and C. Bolm, Chem.-Eur. J., 2014, 20, 4896-4900; (v) K. Yang, Y.-Q. Wang, X.-Y. Chen, A. A. Kadi, H.-K. Fun, H. Sun, Y. Zhang and H.-J. Lu, Chem. Commun., 2015, 51, 3582-3585; (w) C. Wang, C.-P. Chen, J.-Y. Zhang, J. Han, Q. Wang, K. Guo, P. Liu, M.-Y. Guan, Y.-M. Yao and Y.-S. Zhao, Angew. Chem., Int. Ed., 2014, 53, 9884-9888; (x) X.-H. Ye and X.-D. Shi, Org. Lett., 2014, 16, 4448-4451; (y) P. Liu, J. Han, Q. Wang, Z.-B. Huang, D.-Q. Shi, R.-S. Zeng and Y.-S. Zhao, RSC Adv., 2015, 5, 60646-60649; (z) G. Kumar and G. Sekar, RSC Adv., 2015, 5, 28292-28298.

3 Recent reviews for directing group assisted $\mathrm{C}-\mathrm{H}$ functionalization: (a) R.-Y. Zhu, M.-E. Farmer, Y.-Q. Chen and J.Q. Yu, Angew. Chem., Int. Ed., 2016, 55, 10578-10599; (b) D. A. Colby, A. S. Tsai, R. G. Bergman and J. A. Ellman, Acc. Chem. Res., 2011, 45, 814-825; (c) J. J. Mousseau and A. B. Charette, Acc. Chem. Res., 2013, 46, 412-424; (d) R. Giri, B.-F. Shi, K. M. Engle, N. Maugel and J.-Q. Yu, Chem. Soc. Rev., 2009, 38, 3242-3272; (e) A. Ros, R. Fernandez and J. M. Lassaletta, Chem. Soc. Rev., 2014, 43, 
3229-3243; (f) J.-D. Liu, G.-S. Chen and Z. Tan, Adv. Synth. Catal., 2016, 358, 1174-1194; $(g)$ C. S. Yeung and V. M. Dong, Chem. Rev., 2011, 111, 1215-1292; (h) G. Rousseau and B. Breit, Angew. Chem., Int. Ed., 2011, 50, 2450-2494; (i) L. Ackermann, Acc. Chem. Res., 2014, 47, 281295; (j) A. P. Pulis and D. J. Procter, Angew. Chem., Int. Ed., 2016, 55, 9842-9860; (k) J. L. Roizen, M. E. Harvey and J. D. Bois, Acc. Chem. Res., 2012, 45, 911-922; (l) L. Ackermann, Chem. Rev., 2011, 111, 1315-1345; (m) C. Zheng and S.-L. You, RSC Adv., 2014, 4, 6173-6214; (n) G.-F. Zha, H.-L. Qin and E. A. B. Kantchev, RSC Adv., 2016, 6, 30875-30885; (o) T. S. Lobana, RSC Adv., 2015, 5, 3723137274; (p) S. E. Kazzouli, J. Koubachi, N. E. Brahmia and G. Guillaumet, RSC Adv., 2015, 5, 15292-15327; (q) M. Zhang, Y. Zhang, X. Jie, H. Zhao, G. Li and W. Su, Org. Chem. Front., 2014, 1, 843-895; (r) G. Qiu and J. Wu, Org. Chem. Front., 2015, 2, 169-178.

4 Selected papers for directing group assisted $\mathrm{C}-\mathrm{H}$ functionalization: (a) Y.-Q. Yang, X.-D. Qiu, Y. Zhao, Y.-C. Mu and Z.-Z. Shi, J. Am. Chem. Soc., 2016, 138, 495498; (b) K. L. Hull, E. L. Lanni and M. S. Sanford, J. Am. Chem. Soc., 2006, 128, 14047-14049; (c) L. V. Desai, K. L. Hull and M. S. Sanford, J. Am. Chem. Soc., 2004, 126, 9542-9543; (d) M. Yu, Y.-J. Xie, C.-S. Xie and Y.-H. Zhang, Org. Lett., 2012, 14, 2164-2167; (e) X. Chen, C. E. Goodhue and J.-Q. Yu, J. Am. Chem. Soc., 2006, 128, 12634-12635; (f) Y.-J. Liu, Y.-H. Liu, Z.-Z. Zhang, S.-Y. Yan, K. Chen and B.-F. Shi, Angew. Chem., Int. Ed., 2016, 128, 14063-14066; $(g)$ L. Ackermann, A. Althammer and R. Born, Angew. Chem., Int. Ed., 2006, 45, 2619-2622; (h) C.-H. Huang, B. Chattopadhyay and V. Gevorgyan, J. Am. Chem. Soc., 2011, 133, 12406-12409; (i) X.-B. Wan, Z.-X. Ma, B.-J. Li, K.-Y. Zhang, S.-K. Cao, S.-W. Zhang and Z.-J. Shi, J. Am. Chem. Soc., 2006, 128, 7416-7417; (j) T. Kang, Y. Kim, D. Lee, Z. Wang and S. Chang, J. Am. Chem. Soc., 2014, 136, 4141-4144; (k) M. Nishino, K. Hirano, T. Satoh and M. Miura, Angew. Chem., Int. Ed., 2013, 52, 4457-4461; (l) O. Daugulis and V. G. Zaitsev, Angew. Chem., Int. Ed., 2005, 44, 4046-4048; $(m)$ A. R. Dick, K. L. Hull and M. S. Sanford, J. Am. Chem. Soc., 2004, 126, 2300-2301; (n) D.-D. Li, T.-T. Yuan and G.-W. Wang, Chem. Commun., 2011, 47, 12789-12791; (o) C.-M. Wang, H. Chen, Z.-F. Wang, J.-A. Chen and Y. Huang, Angew. Chem., Int. Ed., 2012, 51, 7242-7245; (p) M. Wasa, K. M. Engle and J.-Q. Yu, J. Am. Chem. Soc., 2009, 131, 9886-9887; $(q)$ Z. Ren, F. Mo and G. Dong, J. Am. Chem. Soc., 2012, 134, 16991-16994; $(r)$ M. Mewald, J. A. Schiffner and M. Oestreich, Angew. Chem., Int. Ed., 2012, 51, 1763-1765; (s) H.-Y. Thu, W.-Y. Yu and C.-M. Che, J. Am. Chem. Soc., 2006, 128, 9048-9049; $(t)$ G.-X. Cai, Y. Fu, Y.-Z. Li, X.-B. Wan and Z.-J. Shi, J. Am. Chem. Soc., 2007, 129, 7666-7673; (u) A. García-Rubia, R. G. Arrayás and J. C. Carretero, Angew. Chem., Int. Ed.,
2009, 48, 6511-6515; (v) Z.-L. Fan, J.-B. Ni and A. Zhang, J. Am. Chem. Soc., 2016, 138, 8470-8475; (w) Y. Ano, M. Tobisu and N. Chatani, J. Am. Chem. Soc., 2011, 133, 12984-12986; $(x)$ B. Liu, R. Li, W. Zhan, X. Wang, Z. Ge and R. Li, RSC $A d v$., 2016, 6, 48205-48211; (y) R. Sunke, V. Kumar, E. V. V. S. Ramarao, R. Bankala, K. V. L. Parsa and M. Pal, RSC Adv., 2015, 5, 70604-70608; (z) C. Geng, M. Jiang, L. Feng and P. Jiao, RSC Adv., 2016, 6, 56971-56976.

5 (a) C. T. Walsh, R. V. O'Brien and C. Khosla, Angew. Chem., Int. Ed., 2013, 52, 7098-7124; (b) H. B. Kroona, N. L. Peterson, J. F. Koerner and R. L. Johnson, J. Med. Chem., 1991, 34, 1692-1699; (c) J. S. R. Kumar and A. Datta, Tetrahedron Lett., 1997, 38, 473-476; (d) F. Schweizer, Angew. Chem., Int. Ed., 2002, 41, 230-253; (e) S. Y. Kwak, J. K. Yang, J. H. Kim and Y. S. Lee, Biopolymers, 2013, 100, 584-591; $(f)$ E. Gershonov, R. Granoth, E. Tzehoval, Y. Gaoni and M. Fridkin, J. Med. Chem., 1996, 39, 4833-4843; (g) Y. Wang, J. B. Gloer, J. A. Scott and D. Malloch, J. Nat. Prod., 1995, 58, 93-99.

6 For selected reviews and papers: (a) G. He, B. Wang, W. A. Nack and G. Chen, Acc. Chem. Res., 2016, 49, 635-645; (b) M.-Y. Fan and D.-W. Ma, Angew. Chem., Int. Ed., 2013, 52, 12152-12155; (c) B. D. Dangel, J. A. Johnson and D. Sames, J. Am. Chem. Soc., 2001, 123, 8149-8150; (d) B. Wang, W. A. Nack, G. He, S.-Y. Zhang and G. Chen, Chem. Sci., 2014, 5, 3952-3957; (e) S. Aspin, A. S. Goutierre, P. Larini, R. Jazzar and O. Baudoin, Angew. Chem., Int. Ed., 2012, 51, 10808-10811; (f) B. V. S. Reddy, L. R. Reddy and E. J. Corey, Org. Lett., 2006, 8, 3391-3394; (g) S.-Y. Zhang, Q. Li, G. He, W. A. Nack and G. Chen, J. Am. Chem. Soc., 2013, 135, 12135-12141; (h) J. He, S. Li, Y. Deng, H. Fu, B. N. Laforteza, J. E. Spangler, A. Homs and J.-Q. Yu, Science, 2014, 343, 1216-1220; (i) Q. Zhang, K. Chen, W.-H. Rao, Y. Zhang, F.-J. Chen and B.-F. Shi, Angew. Chem., Int. Ed., 2013, 52, 13588-13592; (j) G.-F. Zhang, X.-Q. Xie, J.-F. Zhu, S.-S. Li, C.-R. Ding and P. Ding, Org. Biomol. Chem., 2015, 13, 5444-5449; (k) L. D. Tran and O. Daugulis, Angew. Chem., Int. Ed., 2012, 51, 5188-5191; (l) F. Gao, W. Zhu, D.-Y. Zhang, S.-J. Li, J. Wang and H. Liu, J. Org. Chem., 2016, 81, 9122-9130; (m) R.-Y. Zhu, K. Tanaka, G.-C. Li, J. He, H.-Y. Fu, S.-H. Li and J.-Q. Yu, J. Am. Chem. Soc., 2015, 137, 7067-7070; (n) N. Rodriguez, J. A. RomeroRevilla, M. A. Fernandez-Ibanez and J. C. Carretero, Chem. Sci., 2013, 4, 175-179; (o) K. Chen, F. Hu, S.-Q. Zhang and B.-F. Shi, Chem. Sci., 2013, 4, 3906-3911; (p) J. Kim, M. Sim, N. Kim and S. Hong, Chem. Sci., 2015, 6, 3611-3616; $(q)$ F.-M. Meyer, S. Liras, A. Guzman-Perez, C. Perreault, J.-W. Bian and K. James, Org. Lett., 2010, 12, 3870-3873.

7 (a) J.-J. Li, T.-S. Mei and J.-Q. Yu, Angew. Chem., Int. Ed., 2008, 47, 6452-6455; (b) A. García-Rubia, E. Laga, C. Cativiela, E. P. Urriolabeitia, R. Gómez-Arrayás and J. C. Carretero, J. Org. Chem., 2015, 80, 3321-3331.

8 Y.-S. Zhao and G. Chen, Org. Lett., 2011, 13, 4850-4853. 